DDS - Die Deutsche Schule 113. Jahrgang 2020, Heft 4, Anhang S. 1-23 https://doi.org/10.31244/dds.2021.04.10

(C) 2021 Waxmann

Julia Hugo

\title{
Verwendete Normen zum Themenschwerpunkt: Chancenungleichheit aus bildungsrechtlicher Perspektive. Inklusion, Übergänge und Homeschooling im Fokus
}

Bei den nachfolgenden Regelungstexten handelt es sich um nicht-amtliche Lesefassungen zum Zeitpunkt der Recherche für das Heft 4/2021 der Zeitschrift DDS - Die Deutsche Schule (Stand 15.10.2021) und erhebt keinen Anspruch auf Vollständigkeit. Fehler sind nicht ausgeschlossen. Es wird ausdrücklich darauf hingewiesen, dass aufgrund möglicher rechtlicher Novellierungen stets die aktuelle, im Gesetz- und Verordnungsblatt veröffentlichte Fassung der jeweiligen Regelung maßgeblich ist. 


\section{Inhaltsverzeichnis}

Allgemeine Erklärung der Menschenrechte (AEMR)

Bayerisches Gesetz über das Erziehungs- und Unterrichtswesen

Bayerische Realschulordnung (RSO) und Gymnasialschulordnung (GSO)

Beamtenstatusgesetz (BeamtStG)

Brandenburgisches Schulgesetz (BbgSchulG) und Verordnung über die Bildungsgänge in der

Sekundarstufe I (Bbg Sek I-VO)

Bremisches Schulgesetz (BremSchulG) und Verordnung über die Organisation des Bildungsgangs der

Grundschule (Grundschulverordnung) im Land Bremen (GrSchulOrgV BR)

Bürgerliches Gesetzbuch (BGB)

Bundesverfassungsgerichtsgesetz (BVerfGG)

Europäische Menschenrechtskonvention (EMRK)

Grundgesetz (GG)

Hamburgisches Schulgesetz (HmbSG)

Hessisches Schulgesetz (HSchG)

Infektionsschutzgesetz (IfSG)

Niedersächsisches Schulgesetz (NSchG)

Sächsisches Schulgesetz (SächsSchG) und Schulordnung Gymnasien Abiturprüfung (SächsSOGYA)

Schleswig-Holsteinisches Schulgesetz (SchulG SH)

Schulgesetz für Baden-Württemberg (SchG B-W) und Aufnahmeverordnung (AufnV BW)

Schulgesetz für das Land Mecklenburg-Vorpommern (SchulG M-V)

Schulgesetz für das Land Nordrhein-Westfalen (NRW - SchulG)

Schulgesetz für das Land Sachsen-Anhalt (SchulG LSA) und Verordnung über die Übergänge zwischen den Schulformen in der Sekundarstufe I

Sozialgesetzbuch (SGB)

Strafgesetzbuch (StGB)

Thüringer Schulgesetz (ThürSchulG) und Thüringer Schulordnung (ThürSchulO)

Übergreifende Schulordnung Rheinland-Pfalz ÜSchulO Rh.-Pf.

UN-Behindertenrechtskonvention (UN-BRK)

UN-Kinderrechtskonvention (UN-KRK)

Verfassung des Freistaats Bayern (BayVerf)

Verfassung des Freistaats Sachsen (SächsLV)

Verwaltungsgerichtsordnung (VwGO) 


\begin{tabular}{|l|l|}
\hline Norm & Text \\
\hline Art. 26 & (1) Jeder hat das Recht auf Bildung. Die Bildung ist unentgeltlich, zum mindesten der \\
& Grundschulunterricht und die grundlegende Bildung. Der Grundschulunterricht ist obligatorisch. Fach- \\
& und Berufsschulunterricht müssen allgemein verfügbar gemacht werden, und der Hochschulunterricht \\
& muß allen gleichermaßen entsprechend ihren Fähigkeiten offenstehen. \\
& (2) Die Bildung muß auf die volle Entfaltung der menschlichen Persönlichkeit und auf die Stärkung der \\
& Achtung vor den Menschenrechten und Grundfreiheiten gerichtet sein. Sie muß zu Verständnis, Toleranz \\
& $\begin{array}{l}\text { und Freundschaft zwischen allen Nationen und allen rassischen oder religiösen Gruppen beitragen und } \\
\text { der Tätigkeit der Vereinten Nationen für die Wahrung des Friedens förderlich sein. } \\
\text { (3) Die Eltern haben ein vorrangiges Recht, die Art der Bildung zu wählen, die ihren Kindern zuteil werden } \\
\text { soll. } \\
\text { Link: https://www.menschenrechtserklaerung.de/die-allgemeine-erklaerung-der-menschenrechte-3157/ }\end{array}$ \\
\hline
\end{tabular}

Bayerisches Gesetz über das Erziehungs- und Unterrichtswesen

\begin{tabular}{|c|c|}
\hline Norm & Text \\
\hline Art. 44 & $\begin{array}{l}\text { (1) }{ }^{1} \text { Soweit nicht Pflichtschulen zu besuchen sind, haben die Erziehungsberechtigten und die volljährigen } \\
\text { Schülerinnen und Schüler das Recht, Schulart, Ausbildungsrichtung und Fachrichtung zu wählen. }{ }^{2} \text { Für } \\
\text { die Aufnahme sind Eignung und Leistung der Schülerin bzw. des Schülers maßgebend. } \\
\text { (2) }{ }^{1} \text { Für Schulen, die nicht Pflichtschulen sind, wird das zuständige Staatsministerium ermächtigt, die } \\
\text { Voraussetzungen der Aufnahme (einschließlich Altersgrenzen) und eine Probezeit in der Schulordnung } \\
\text { zu regeln; dabei kann die Aufnahme von einer der Aufgabenstellung der Schule entsprechenden } \\
\text { Leistungsfeststellung abhängig gemacht werden. }{ }^{2} \text { Ab Jahrgangsstufe } 10 \text { kann die Aufnahme versagt } \\
\text { werden, wenn die Schülerin oder der Schüler wegen einer vorsätzlich begangenen Straftat zu einer } \\
\text { Freiheitsstrafe von mindestens einem Jahr rechtskräftig verurteilt worden ist, die Strafe noch der } \\
\text { unbeschränkten Auskunft unterliegt und wenn nach der Art der begangenen Straftat durch die } \\
\text { Anwesenheit des Schülers die Sicherheit oder die Ordnung des Schulbetriebs oder die Verwirklichung } \\
\text { der Bildungsziele der Schule erheblich gefährdet wäre. } \\
\text { (3) Ein Rechtsanspruch auf Aufnahme in eine bestimmte Schule an einem bestimmten Ort besteht nicht. } \\
\text { (4) }{ }^{1} \text { Die Zulassung zu einer Ausbildungs- oder Fachrichtung einer Schulart darf im notwendigen Umfang } \\
\text { nur dann beschränkt werden, wenn die Zahl der Bewerbungen die Zahl der Ausbildungsplätze erheblich } \\
\text { übersteigt und ein geordneter Unterrichtsbetrieb nicht mehr sichergestellt werden kann. 2Das zuständige } \\
\text { Staatsministerium wird ermächtigt, im Benehmen mit dem Landesschulbeirat durch Rechtsverordnung } \\
\text { das Verfahren der Zulassung nach Gesichtspunkten der Eignung und der Leistung zu regeln; Wartezeit } \\
\text { und Härtefälle sollen berücksichtigt werden; für kommunale Schulen kann der Schulträger dies durch } \\
\text { eine Satzung regeln, falls eine Rechtsverordnung für die betreffende Schulart und Ausbildungsrichtung } \\
\text { nicht erlassen worden ist. } \\
\text { Link: https://www.gesetze-bayern.de/Content/Document/BayEUG-44 }\end{array}$ \\
\hline
\end{tabular}

Bayerische Realschulordnung (RSO) und Gymnasialschulordnung (GSO)

\begin{tabular}{|c|c|}
\hline Norm & Text \\
\hline $\begin{array}{l}\text { § } 2 \\
\text { BayRS } \\
0\end{array}$ & $\begin{array}{l}\text { (1) Die Schülerinnen und Schüler werden von einem Erziehungsberechtigten angemeldet. } \\
\text { (2) Die Aufnahme in die unterste Jahrgangsstufe setzt voraus, dass die Schülerin oder der Schüler } \\
\text { 1. für den Bildungsweg der Realschule geeignet ist, } \\
\text { 2. am 30. September des Schuljahres das 12. Lebensjahr noch nicht vollendet hat; über } \\
\text { Ausnahmen in besonderen Fällen entscheidet die Schulleiterin oder der Schulleiter. } \\
\text { (3) Für den Bildungsweg der Realschule sind geeignet } \\
\text { 1. Schülerinnen und Schüler einer öffentlichen oder staatlich anerkannten Grundschule, wenn sie } \\
\text { im Übertrittszeugnis dieser Schule oder im Jahreszeugnis der Jahrgangsstufe } 5 \text { einer } \\
\text { öffentlichen oder staatlich anerkannten Mittelschule als geeignet für den Bildungsweg der } \\
\text { Realschule oder des Gymnasiums bezeichnet sind, } \\
\text { 2. Schülerinnen und Schüler, die mit Erfolg am Probeunterricht teilgenommen haben, } \\
\text { 3. Schülerinnen und Schüler einer öffentlichen oder staatlich anerkannten Grundschule, denen } \\
\text { zum Schulhalbjahr, d.h. zum letzten Unterrichtstag der zweiten vollen Unterrichtswoche im } \\
\quad \text { Februar, oder zum Ende der Jahrgangsstufe } 3 \text { das Überspringen der Jahrgangsstufe } 4 \text { gestattet } \\
\text { worden ist, } \\
\text { 4. Schülerinnen und Schüler eines öffentlichen oder staatlich anerkannten Gymnasiums, wenn sie } \\
\text { nicht dem Wiederholungsverbot nach Art. } 53 \text { Abs. } 3 \text { BayEUG unterliegen. } \\
\text { (4) Es werden auch Schülerinnen und Schüler aufgenommen, die in beiden Fächern nur mit der Note } 4 \\
\text { und damit ohne Erfolg am Probeunterricht der Realschule oder des Gymnasiums teilgenommen haben, } \\
\text { deren Erziehungsberechtigte aber die Aufnahme gleichwohl beantragen. } \\
\text { (5) Das Übertrittszeugnis, der mit Erfolg besuchte Probeunterricht, die Entscheidung über das } \\
\text { Überspringen und das Zeugnis des Gymnasiums gelten nur für den Übertritt an die Realschule im } \\
\text { folgenden Schuljahr. } \\
\text { (6) An öffentlichen Heimschulen kann die Aufnahme von Externen auf Schülerinnen und Schüler } \\
\text { beschränkt werden, die ihren Wohnsitz im Sinn des Bürgerlichen Gesetzbuchs im Einzugsbereich der } \\
\text { Schule haben. }\end{array}$ \\
\hline
\end{tabular}




\begin{tabular}{|c|c|}
\hline & $\begin{array}{l}\text { (7) }{ }^{1} \text { Sind mehr Bewerberinnen und Bewerber vorhanden als im Hinblick auf die räumlichen und } \\
\text { personellen Verhältnisse der Schule aufgenommen werden können, so bemühen sich die Leiterinnen } \\
\text { oder Leiter der staatlichen und nicht staatlichen Schulen um einen örtlichen Ausgleich. }{ }^{2} \text { Gelingt dieser } \\
\text { nicht, so entscheidet die oder der Ministerialbeauftragte mit Wirkung für die öffentlichen Schulen. } \\
\text { (8) Die Aufnahme erfolgt zu Beginn des Schuljahres, sonst nur aus wichtigem Grund. } \\
\text { Link: https://www.gesetze-bayern.de/Content/Document/BayRSO-2 }\end{array}$ \\
\hline $\begin{array}{l}\S 2 \\
\text { BayGS } \\
0\end{array}$ & $\begin{array}{l}\text { (1) }{ }^{1} \text { Für Schülerinnen und Schüler, bei denen die Voraussetzungen nach } \S 2 \text { Abs. } 3 \text { Satz } 1 \text { Nr. } 1 \text { oder } 3 \\
\text { nicht gegeben sind und die nicht der Jahrgangsstufe } 5 \text { einer öffentlichen oder staatlich anerkannten } \\
\text { Mittel- oder Realschule angehören, führen Gymnasien nach den Vorgaben der Ministerialbeauftragten } \\
\text { einen dreitägigen Probeunterricht in den Fächern Deutsch und Mathematik durch. 2In begründeten } \\
\text { Ausnahmefällen kann ein Nachholtermin für den Probeunterricht eingerichtet werden. }{ }^{3} \text { Schülerinnen und } \\
\text { Schüler, die ohne Erfolg am Probeunterricht der Realschule teilgenommen haben, können nicht am } \\
\text { Probeunterricht des Gymnasiums teilnehmen. } \\
\text { (2) Für die Vorbereitung und Durchführung des Probeunterrichts beruft die Schulleiterin oder der } \\
\text { Schulleiter einen Aufnahmeausschuss ein, der sich aus Lehrkräften des Gymnasiums zusammensetzt. } \\
\text { (3) } 1 \text { Im Probeunterricht sollen die Schülerinnen und Schüler in kleineren Unterrichtsgruppen } \\
\text { zusammengefasst werden. }{ }^{2} \text { Für jede Unterrichtsgruppe sind mindestens zwei Mitglieder des } \\
\text { Aufnahmeausschusses verantwortlich, die abwechselnd unterrichten und beobachten. }{ }^{3} \text { Dem } \\
\text { Probeunterricht werden die Anforderungen der Jahrgangsstufe } 4 \text { unter Berücksichtigung der Aufgabe } \\
\text { des Gymnasiums zugrunde gelegt. } \\
\text { (4) }{ }^{1} \text { Die schriftlichen Aufgaben werden landeseinheitlich gestellt und von je zwei Fachlehrkräften benotet; } \\
\text { die Arbeiten sind zwei Jahre aufzubewahren. }{ }^{2} \text { Auch die mündlichen Leistungen werden benotet. } \\
\text { (5) 'Die Teilnahme am Probeunterricht ist erfolgreich, wenn in dem einen Fach mindestens die Note } 3 \\
\text { und in dem anderen Fach mindestens die Note } 4 \text { erreicht wurde. }{ }^{2} \text { Die erfolglose Teilnahme wird auf dem } \\
\text { Übertrittszeugnis der Grundschule vermerkt. }{ }^{3} \text { Werden die Schülerinnen und Schüler nicht } \\
\text { aufgenommen, erhalten die Erziehungsberechtigten das Übertrittszeugnis zurück. } \\
\text { Link: https://www.gesetze-bayern.de/Content/Document/BayGSO-3 }\end{array}$ \\
\hline
\end{tabular}

Beamtenstatusgesetz (BeamtStG)

\begin{tabular}{|l|l|}
\hline Norm & Text \\
\hline Art. 36 & (2) Bedenken gegen die Rechtmäßigkeit dienstlicher Anordnungen haben Beamtinnen und Beamte \\
Abs. 2 & unverzüglich auf dem Dienstweg geltend zu machen. Wird die Anordnung aufrechterhalten, haben sie \\
& sich, wenn die Bedenken fortbestehen, an die nächst höhere Vorgesetzte oder den nächst höheren \\
& Vorgesetzten zu wenden. Wird die Anordnung bestätigt, müssen die Beamtinnen und Beamten sie \\
& ausführen und sind von der eigenen Verantwortung befreit. Dies gilt nicht, wenn das aufgetragene \\
& $\begin{array}{l}\text { Verhalten die Würde des Menschen verletzt oder strafbar oder ordnungswidrig ist und die Strafbarkeit } \\
\text { oder Ordnungswidrigkeit für die Beamtinnen oder Beamten erkennbar ist. Die Bestätigung hat auf }\end{array}$ \\
& Verlangen schriftlich zu erfolgen. \\
& Link: https://www.gesetze-im-internet.de/beamtstg/
\end{tabular}

Brandenburgisches Schulgesetz (BbgSchulG) und Verordnung über die Bildungsgänge in der Sekundarstufe I (Bbg Sek I-VO)

\begin{tabular}{|c|c|}
\hline Norm & Text \\
\hline$\S 53$ & $\begin{array}{l}\text { (1) Für die Aufnahme in eine weiterführende allgemein bildende Schule sind neben dem Wunsch der } \\
\text { Eltern die Fähigkeiten, Leistungen und Neigungen (Eignung) der Schülerin oder des Schülers } \\
\text { maßgebend. Die Eltern wählen durch einen Erstwunsch und einen Zweitwunsch je eine Schule, an der } \\
\text { ihr Kind den gewünschten Bildungsgang belegen soll. } \\
\text { (2) Die Eltern sind durch die Lehrkräfte der weiterführenden allgemein bildenden Schule über das } \\
\text { Auswahlverfahren bei Übernachfrage, die Abschlüsse und Berechtigungen der Bildungsgänge der } \\
\text { Sekundarstufe I und die sich daraus jeweils ergebenden Möglichkeiten der Fortsetzung der Ausbildung } \\
\text { in der Sekundarstufe II sowie über die jeweiligen Bildungsziele des gewählten Bildungsgangs zu beraten. } \\
\text { Dabei ist insbesondere auf die Bedeutung der Fremdsprachenfolge einzugehen. } \\
\text { (3) Der Besuch eines Bildungsgangs setzt die dafür erforderliche Eignung voraus. Übersteigt die Zahl } \\
\text { der Anmeldungen für eine Schule die Aufnahmekapazität, wird ein Auswahlverfahren durchgeführt. Die } \\
\text { Auswahl erfolgt an Gymnasien nach } \\
\text { 1. besonderen Härtefällen gemäß Absatz } 4 \text {, } \\
\text { 2. dem Vorrang der Eignung gemäß Absatz } 5 \text { und } \\
3 \text {. dem Vorliegen besonderer Gründe. } \\
\text { Das Vorliegen eines besonderen Grundes rechtfertigt den Vorrang einer Schülerin oder eines Schülers } \\
\text { bei gleicher Eignung für den Bildungsgang in der gewählten Schule. Die Auswahl erfolgt an Oberschulen } \\
1 . \quad \text { nach besonderen Härtefällen gemäß Absatz } 4 \text { und } \\
2 . \text { im Übrigen nach der Nähe der Wohnung zur Schule. } \\
\text { Im Umfang von bis zu } 50 \text { vom Hundert der Aufnahmekapazität können Schülerinnen und Schüler } \\
\text { vorrangig berücksichtigt werden, wenn ein besonderer Grund vorliegt. An Gesamtschulen erfolgt die } \\
\text { Aufnahme zu einem Drittel der Aufnahmekapazität für den Bildungsgang zum Erwerb der allgemeinen } \\
\text { Hochschulreife nach dem Vorrang der Eignung gemäß Absatz } 5 \text { Satz } 4 \text { bis } 6 \text { und zu zwei Dritteln der } \\
\text { Aufnahmekapazität entsprechend dem Aufnahmeverfahren an Oberschulen. }\end{array}$ \\
\hline
\end{tabular}

DDS, 113. Jg., 4(2021) 


\begin{tabular}{|c|c|}
\hline & 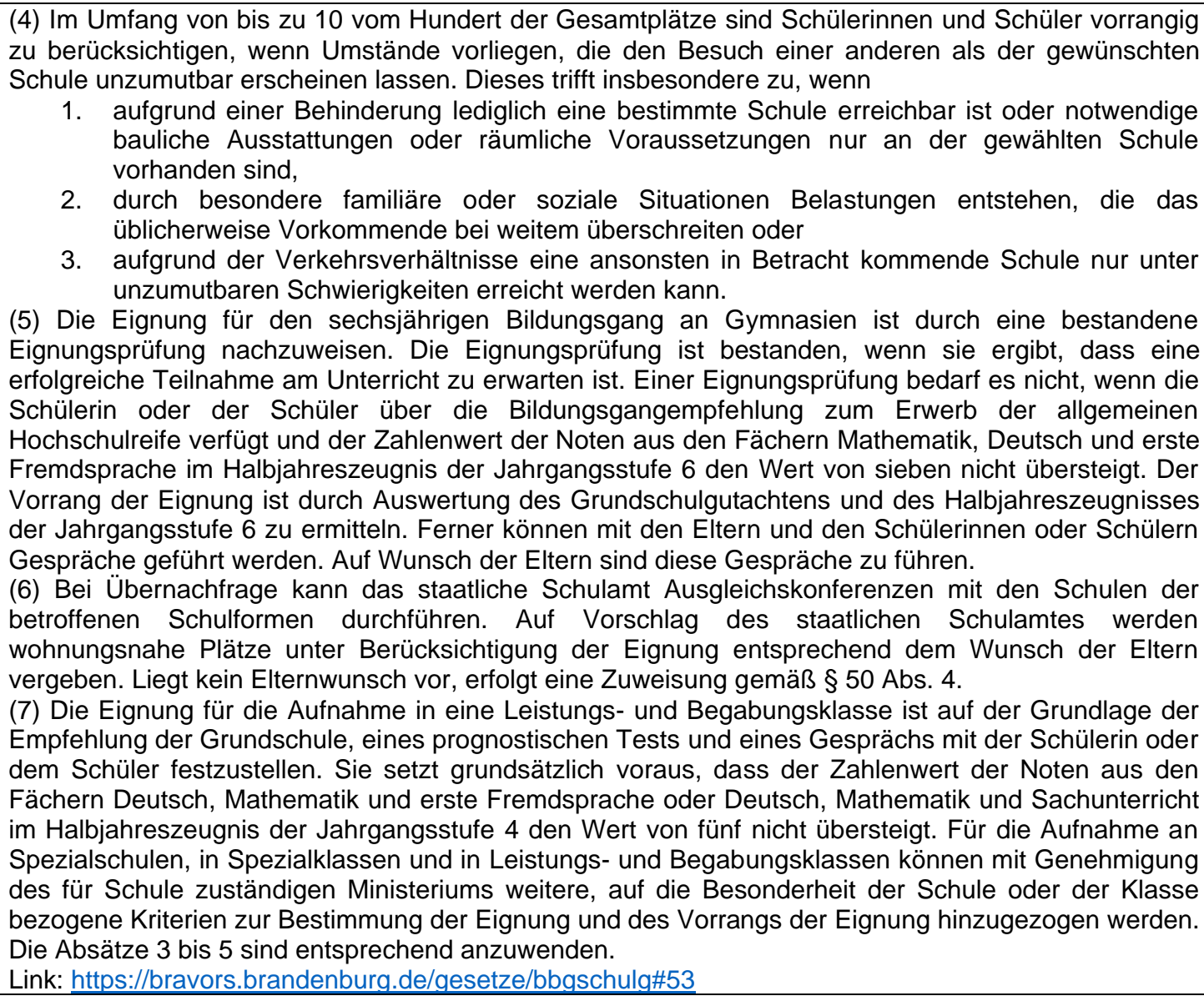 \\
\hline $\begin{array}{l}\S 40 \\
\text { Bbg. } \\
\text { Sek I- } \\
\text { VO }\end{array}$ & $\begin{array}{l}\text { (1) Das Aufnahmeverfahren in die Jahrgangsstufe } 7 \text { besteht aus } \\
\text { 1. der Eignungsfeststellung gemäß } \S 41 \text { und } \S 42 \text {, } \\
\text { 2. dem Auswahlverfahren gemäß } \$ 43 \text { und } \S 7 \text { und } \\
\text { 3. gegebenenfalls dem Zuweisungsverfahren gemäß } \S 7 \text {. } \\
\text { (2) Die Durchführung der Eignungsfeststellung und des Auswahlverfahrens obliegt den Schulleiterinnen } \\
\text { und Schulleitern der gewünschten Schulen. } \\
\text { (3) Die Durchführung des Zuweisungsverfahrens obliegt dem staatlichen Schulamt. Die Schülerinnen } \\
\text { und Schüler, die nach der Eignungsfeststellung und dem Auswahlverfahren keine Aufnahme finden, } \\
\text { nehmen am Zuweisungsverfahren teil. } \\
\text { Link: https://bravors.brandenburg.de/verordnungen/sek i v\#40 }\end{array}$ \\
\hline
\end{tabular}

Bremisches Schulgesetz (BremSchulG) und Verordnung über die Organisation des Bildungsgangs der Grundschule (Grundschulverordnung) im Land Bremen (GrSchulOrgV BR)

\begin{tabular}{|c|c|}
\hline Norm & Text \\
\hline $\begin{array}{l}\$ 37 a \\
\text { BremS } \\
\text { chulG }\end{array}$ & $\begin{array}{l}\text { Am Ende des Bildungsganges der Grundschule wählen die Erziehungsberechtigten nach Beratung } \\
\text { durch die Grundschule den weiteren Bildungsgang für ihr Kind. Nehmen die Erziehungsberechtigten } \\
\text { nicht an der Beratung teil, weist die Grundschule die Schülerin oder den Schüler einer Schulart zu. Die } \\
\text { Aufnahme an der jeweiligen Schule erfolgt nach } \S \S 6 \text { bis } 6 b \text { des Bremischen Schulverwaltungsgesetzes. } \\
\text { Link: https://www.transparenz.bremen.de/metainformationen/bremisches-schulgesetz-bremschulg-in- } \\
\text { der-fassung-der-bekanntmachung-vom-28-juni-2005- } \\
149151 \text { ?asl=bremen02.c.732.de\&template=20 gp ifg meta detail d\#ilr-SchulGBRV4P37a }\end{array}$ \\
\hline $\begin{array}{l}\text { § } 13 \\
\text { GrSchu } \\
\text { IOrgV } \\
\text { BR }\end{array}$ & $\begin{array}{l}\text { (1) Zum Ende des ersten Halbjahres der 4. Jahrgangsstufe erhalten die Schülerinnen und Schüler, } \\
\text { zusätzlich zum Zeugnis oder zum Lernentwicklungsbericht, eine Bewertung ihrer Leistungen in den } \\
\text { Kompetenzbereichen der Bildungsstandards in Deutsch und Mathematik. } \\
\text { (2) Die Zeugniskonferenz legt fest, ob die Leistungen in allen Kompetenzbereichen in Deutsch und } \\
\text { Mathematik über dem Regelstandard liegen oder nicht. } \\
\text { (3) In einem verbindlichen Beratungsgespräch über den weiteren Bildungsgang ihres Kindes werden } \\
\text { den Erziehungsberechtigten die Leistungen erläutert. } \\
\text { (4) Die enge Zusammenarbeit zwischen der Grundschule und den regionalen weiterführenden Schulen } \\
\text { ist Voraussetzung für einen kontinuierlichen Bildungsweg der Schülerinnen und Schüler. Zur } \\
\text { Abstimmung und Koordinierung des Übergangs führen die Schulen gemeinsame Dienstbesprechungen } \\
\text { durch. Dabei sollen Strukturen geschaffen werden, die die durchgängige Förderung und Forderung der } \\
\text { Schülerinnen und Schüler ermöglichen. }\end{array}$ \\
\hline
\end{tabular}


Link: https://www.transparenz.bremen.de/metainformationen/verordnung-ueber-die-organisation-desbildungsgangs-der-grundschule-grundschulverordnung-im-land-bremen-vom-1-august-201267033?as|=bremen203 tpgesetz.c.55340.de\&template=20 gp ifg meta detail d\#

Bürgerliches Gesetzbuch (BGB)

\begin{tabular}{|c|c|}
\hline Norm & Text \\
\hline $\begin{array}{l}\S 1666 \\
\text { Abs. } 1\end{array}$ & $\begin{array}{l}\text { (1) Wird das körperliche, geistige oder seelische Wohl des Kindes oder sein Vermögen gefährdet und } \\
\text { sind die Eltern nicht gewillt oder nicht in der Lage, die Gefahr abzuwenden, so hat das Familiengericht } \\
\text { die Maßnahmen zu treffen, die zur Abwendung der Gefahr erforderlich sind. }\end{array}$ \\
\hline Abs. 3 & $\begin{array}{l}\text { (3) Zu den gerichtlichen Maßnahmen nach Absatz } 1 \text { gehören insbesondere } \\
\text { 1. 1.Gebote, öffentliche Hilfen wie zum Beispiel Leistungen der Kinder- und Jugendhilfe und der } \\
\text { Gesundheitsfürsorge in Anspruch zu nehmen, } \\
\text { 2. 2.Gebote, für die Einhaltung der Schulpflicht zu sorgen, } \\
\text { 3. } \\
\text { 3.Verbote, vorübergehend oder auf unbestimmte Zeit die Familienwohnung oder eine andere } \\
\text { Wohnung zu nutzen, sich in einem bestimmten Umkreis der Wohnung aufzuhalten oder zu } \\
\text { bestimmende andere Orte aufzusuchen, an denen sich das Kind regelmäßig aufhält, } \\
\text { 4. } \\
\text { 4.Verbote, Verbindung zum Kind aufzunehmen oder ein Zusammentreffen mit dem Kind } \\
\text { h. } \\
\text { 5. 5.die Ersetzung von Erklärungen des Inhabers der elterlichen Sorge, } \\
\text { 6. 6.die teilweise oder vollständige Entziehung der elterlichen Sorge. } \\
\text { Link: https://www.gesetze-im-internet.de/bgb/ 1666.html }\end{array}$ \\
\hline $\begin{array}{l}\S \\
1666 a\end{array}$ & $\begin{array}{l}\text { (1) Maßnahmen, mit denen eine Trennung des Kindes von der elterlichen Familie verbunden ist, sind } \\
\text { nur zulässig, wenn der Gefahr nicht auf andere Weise, auch nicht durch öffentliche Hilfen, begegnet } \\
\text { werden kann. Dies gilt auch, wenn einem Elternteil vorübergehend oder auf unbestimmte Zeit die } \\
\text { Nutzung der Familienwohnung untersagt werden soll. Wird einem Elternteil oder einem Dritten die } \\
\text { Nutzung der vom Kind mitbewohnten oder einer anderen Wohnung untersagt, ist bei der Bemessung } \\
\text { der Dauer der Maßnahme auch zu berücksichtigen, ob diesem das Eigentum, das Erbbaurecht oder der } \\
\text { Nießbrauch an dem Grundstück zusteht, auf dem sich die Wohnung befindet; Entsprechendes gilt für } \\
\text { das Wohnungseigentum, das Dauerwohnrecht, das dingliche Wohnrecht oder wenn der Elternteil oder } \\
\text { Dritte Mieter der Wohnung ist. } \\
\text { (2) Die gesamte Personensorge darf nur entzogen werden, wenn andere Maßnahmen erfolglos } \\
\text { geblieben sind oder wenn anzunehmen ist, dass sie zur Abwendung der Gefahr nicht ausreichen. } \\
\text { Link: https://www.gesetze-im-internet.de/bgb/ 1666a.html }\end{array}$ \\
\hline
\end{tabular}

Bundesverfassungsgerichtsgesetz (BVerfGG)

\begin{tabular}{|c|c|}
\hline Norm & Text \\
\hline$\S 93 a$ & $\begin{array}{l}\text { (1) Die Verfassungsbeschwerde bedarf der Annahme zur Entscheidung. } \\
\text { (2) Sie ist zur Entscheidung anzunehmen, } \\
\text { a) soweit ihr grundsätzliche verfassungsrechtliche Bedeutung zukommt, } \\
\text { b) wenn es zur Durchsetzung der in } \S 90 \text { Abs. } 1 \text { genannten Rechte angezeigt ist; dies kann auch } \\
\text { der Fall sein, wenn dem Beschwerdeführer durch die Versagung der Entscheidung zur Sache } \\
\text { ein besonders schwerer Nachteil entsteht. } \\
\text { Link: https://www.gesetze-im-internet.de/bverfgg/ 93a.html }\end{array}$ \\
\hline § 93d & $\begin{array}{l}\text { (1) Die Entscheidung nach § 93b und § 93c ergeht ohne mündliche Verhandlung. Sie ist unanfechtbar. } \\
\text { Die Ablehnung der Annahme der Verfassungsbeschwerde bedarf keiner Begründung. } \\
\text { (2) Solange und soweit der Senat nicht über die Annahme der Verfassungsbeschwerde entschieden hat, } \\
\text { kann die Kammer alle das Verfassungsbeschwerdeverfahren betreffenden Entscheidungen erlassen. } \\
\text { Eine einstweilige Anordnung, mit der die Anwendung eines Gesetzes ganz oder teilweise ausgesetzt } \\
\text { wird, kann nur der Senat treffen; § } 32 \text { Abs. } 7 \text { bleibt unberührt. Der Senat entscheidet auch in den Fällen } \\
\text { des § } 32 \text { Abs. 3. } \\
\text { (3) Die Entscheidungen der Kammer ergehen durch einstimmigen Beschluß. Die Annahme durch den } \\
\text { Senat ist beschlossen, wenn mindestens drei Richter ihr zustimmen. } \\
\text { Link: https://www.gesetze-im-internet.de/bverfgg/ 93d.html }\end{array}$ \\
\hline
\end{tabular}

Europäische Menschenrechtskonvention (EMRK)

\begin{tabular}{|l|l|}
\hline Norm & Text \\
\hline Art. 2 & $\begin{array}{l}\text { (1) Das Recht jedes Menschen auf Leben wird gesetzlich geschützt. Niemand darf absichtlich getötet } \\
\text { werden, außer durch Vollstreckung eines Todesurteils, das ein Gericht wegen eines Verbrechens } \\
\text { verhängt hat, für das die Todesstrafe gesetzlich vorgesehen ist. } \\
\text { Link: https://www.menschenrechtskonvention.eu/konvention-zum-schutz-der-menschenrechte-und- } \\
\text { grundfreiheiten-9236/\#2-artikel-2---recht-auf-leben }\end{array}$ \\
\hline Art. 8 & $\begin{array}{l}\text { (1) Jede Person hat das Recht auf Achtung ihres Privat- und Familienlebens, ihrer Wohnung und ihrer } \\
\text { Korrespondenz. } \\
\text { (2) Eine Behörde darf in die Ausübung dieses Rechts nur eingreifen, soweit der Eingriff gesetzlich } \\
\text { vorgesehen und in einer demokratischen Gesellschaft notwendig ist für die nationale oder öffentliche } \\
\text { Sicherheit, für das wirtschaftliche Wohl des Landes, zur Aufrechterhaltung der Ordnung, zur Verhütung }\end{array}$ \\
\hline
\end{tabular}




\begin{tabular}{|l|l|}
\hline & $\begin{array}{l}\text { von Straftaten, zum Schutz der Gesundheit oder der Moral oder zum Schutz der Rechte und Freiheiten } \\
\text { anderer. } \\
\text { Link: https://www.menschenrechtskonvention.eu/konvention-zum-schutz-der-menschenrechte-und- } \\
\text { grundfreiheiten-9236/\#8-artikel-8-\%E2\%80\%93-recht-auf-achtung-des-privat--und-familienlebens }\end{array}$ \\
\hline Art. 11 & $\begin{array}{l}\text { (1) Jede Person hat das Recht, sich frei und friedlich mit anderen zu versammeln und sich frei mit } \\
\text { anderen zusammenzuschließen; dazu gehört auch das Recht, zum Schutz seiner Interessen } \\
\text { Gewerkschaften zu gründen und Gewerkschaften beizutreten. } \\
\text { (2) Die Ausübung dieser Rechte darf nur Einschränkungen unterworfen werden, die gesetzlich } \\
\text { vorgesehen und in einer demokratischen Gesellschaft notwendig sind für die nationale oder öffentliche } \\
\text { Sicherheit, zur Aufrechterhaltung der Ordnung oder zur Verhütung von Straftaten, zum Schutz der } \\
\text { Gesundheit oder der Moral oder zum Schutz der Rechte und Freiheiten anderer. Dieser Artikel steht } \\
\text { rechtmäßigen Einschränkungen der Ausübung dieser Rechte für Angehörige der Streitkräfte, der Polizei } \\
\text { oder der Staatsverwaltung nicht entgegen. } \\
\text { Link: https://www.menschenrechtskonvention.eu/konvention-zum-schutz-der-menschenrechte-und- } \\
\text { grundfreiheiten-9236/\#11-artikel-11-\%E2\%80\%93-versammlungs--und-vereinigungsfreiheit }\end{array}$ \\
\hline $\begin{array}{l}\text { Art. 2 } \\
\text { erstes } \\
\text { Zusatz } \\
\text { protoko } \\
\text { II }\end{array}$ & $\begin{array}{l}\text { Niemandem darf das Recht auf Bildung verwehrt werden. Der Staat hat bei Ausübung der von ihm auf } \\
\text { dem Gebiet der Erziehung und des Unterrichts übernommenen Aufgaben das Recht der Eltern zu } \\
\text { achten, die Erziehung und den Unterricht entsprechend ihren eigenen religiösen und weltanschaulichen } \\
\text { Uiberzeugungen sicherzustellen. } \\
\text { Link: hiltps://www.menschenrechtskonvention.eu/zusatzprotokoll-emrk-9251/\#1-artikel-2---recht-auf- } \\
\text { bildung }\end{array}$ \\
\hline
\end{tabular}

Grundgesetz (GG)

\begin{tabular}{|c|c|}
\hline Norm & Text \\
\hline $\begin{array}{l}\text { Art. } 1 \\
\text { Abs. } 1\end{array}$ & $\begin{array}{l}\text { (1) Die Würde des Menschen ist unantastbar. Sie zu achten und zu schützen ist Verpflichtung aller } \\
\text { staatlichen Gewalt. } \\
\text { Link: https://www.gesetze-im-internet.de/gg/art 1.html }\end{array}$ \\
\hline $\begin{array}{l}\text { Art. } 2 \\
\text { Abs. } 1\end{array}$ & $\begin{array}{l}\text { (1) Jeder hat das Recht auf die freie Entfaltung seiner Persönlichkeit, soweit er nicht die Rechte anderer } \\
\text { verletzt und nicht gegen die verfassungsmäßige Ordnung oder das Sittengesetz verstößt. } \\
\text { Link: https://www.gesetze-im-internet.de/gg/art 2.html }\end{array}$ \\
\hline Art. 3 & $\begin{array}{l}\text { (1) Alle Menschen sind vor dem Gesetz gleich. } \\
\text { (2) Männer und Frauen sind gleichberechtigt. Der Staat fördert die tatsächliche Durchsetzung der } \\
\text { Gleichberechtigung von Frauen und Männern und wirkt auf die Beseitigung bestehender Nachteile hin. } \\
\text { (3) Niemand darf wegen seines Geschlechtes, seiner Abstammung, seiner Rasse, seiner Sprache, } \\
\text { seiner Heimat und Herkunft, seines Glaubens, seiner religiösen oder politischen Anschauungen } \\
\text { benachteiligt oder bevorzugt werden. Niemand darf wegen seiner Behinderung benachteiligt werden. } \\
\text { Link: https://www.gesetze-im-internet.de/gg/art 3.html }\end{array}$ \\
\hline $\begin{array}{l}\text { Art. } 6 \\
\text { Abs. } 2\end{array}$ & $\begin{array}{l}\text { (2) Pflege und Erziehung der Kinder sind das natürliche Recht der Eltern und die zuvörderst ihnen } \\
\text { obliegende Pflicht. Über ihre Betätigung wacht die staatliche Gemeinschaft. } \\
\text { Link: https://www.gesetze-im-internet.de/gg/art 6.html }\end{array}$ \\
\hline $\begin{array}{l}\text { Art. } 7 \\
\text { Abs. } 1\end{array}$ & $\begin{array}{l}\text { (1) Das gesamte Schulwesen steht unter der Aufsicht des Staates. } \\
\text { Link: https://www.gesetze-im-internet.de/gq/art 7.html }\end{array}$ \\
\hline $\begin{array}{l}\text { Art. } 12 \\
\text { Abs. } 1\end{array}$ & $\begin{array}{l}\text { (1) Alle Deutschen haben das Recht, Beruf, Arbeitsplatz und Ausbildungsstätte frei zu wählen. Die } \\
\text { Berufsausübung kann durch Gesetz oder auf Grund eines Gesetzes geregelt werden. } \\
\text { Link: https://www.gesetze-im-internet.de/gg/art 12.html }\end{array}$ \\
\hline Art. 59 & $\begin{array}{l}\text { (1) Der Bundespräsident vertritt den Bund völkerrechtlich. Er schließt im Namen des Bundes die Verträge } \\
\text { mit auswärtigen Staaten. Er beglaubigt und empfängt die Gesandten. } \\
\text { (2) Verträge, welche die politischen Beziehungen des Bundes regeln oder sich auf Gegenstände der } \\
\text { Bundesgesetzgebung beziehen, bedürfen der Zustimmung oder der Mitwirkung der jeweils für die } \\
\text { Bundesgesetzgebung zuständigen Körperschaften in der Form eines Bundesgesetzes. Für } \\
\text { Verwaltungsabkommen gelten die Vorschriften über die Bundesverwaltung entsprechend. } \\
\text { Link: https://www.gesetze-im-internet.de/gg/art 59.html }\end{array}$ \\
\hline $\begin{array}{l}\text { Art. } 74 \\
\text { Abs. } 1 \\
\text { Nr. } 19\end{array}$ & $\begin{array}{l}\text { (1) Die konkurrierende Gesetzgebung erstreckt sich auf folgende Gebiete: } \\
\text { [...] } \\
\text { 19. Maßnahmen gegen gemeingefährliche oder übertragbare Krankheiten bei Menschen und Tieren, } \\
\text { Zulassung zu ärztlichen und anderen Heilberufen und zum Heilgewerbe, sowie das Recht des } \\
\text { Apothekenwesens, der Arzneien, der Medizinprodukte, der Heilmittel, der Betäubungsmittel und der } \\
\text { Gifte; } \\
\text { Link: https://www.gesetze-im-internet.de/gg/art 74.html }\end{array}$ \\
\hline Art. 94 & $\begin{array}{l}\text { (1) Das Bundesverfassungsgericht besteht aus Bundesrichtern und anderen Mitgliedern. Die Mitglieder } \\
\text { des Bundesverfassungsgerichtes werden je zur Hälfte vom Bundestage und vom Bundesrate gewählt. } \\
\text { Sie dürfen weder dem Bundestage, dem Bundesrate, der Bundesregierung noch entsprechenden } \\
\text { Organen eines Landes angehören. } \\
\text { (2) Ein Bundesgesetz regelt seine Verfassung und das Verfahren und bestimmt, in welchen Fällen seine } \\
\text { Entscheidungen Gesetzeskraft haben. Es kann für Verfassungsbeschwerden die vorherige Erschöpfung } \\
\text { des Rechtsweges zur Voraussetzung machen und ein besonderes Annahmeverfahren vorsehen. } \\
\text { Link: https://www.gesetze-im-internet.de/gg/art_94.html }\end{array}$ \\
\hline
\end{tabular}




\begin{tabular}{|c|c|}
\hline Norm & Text \\
\hline$\S 42$ & 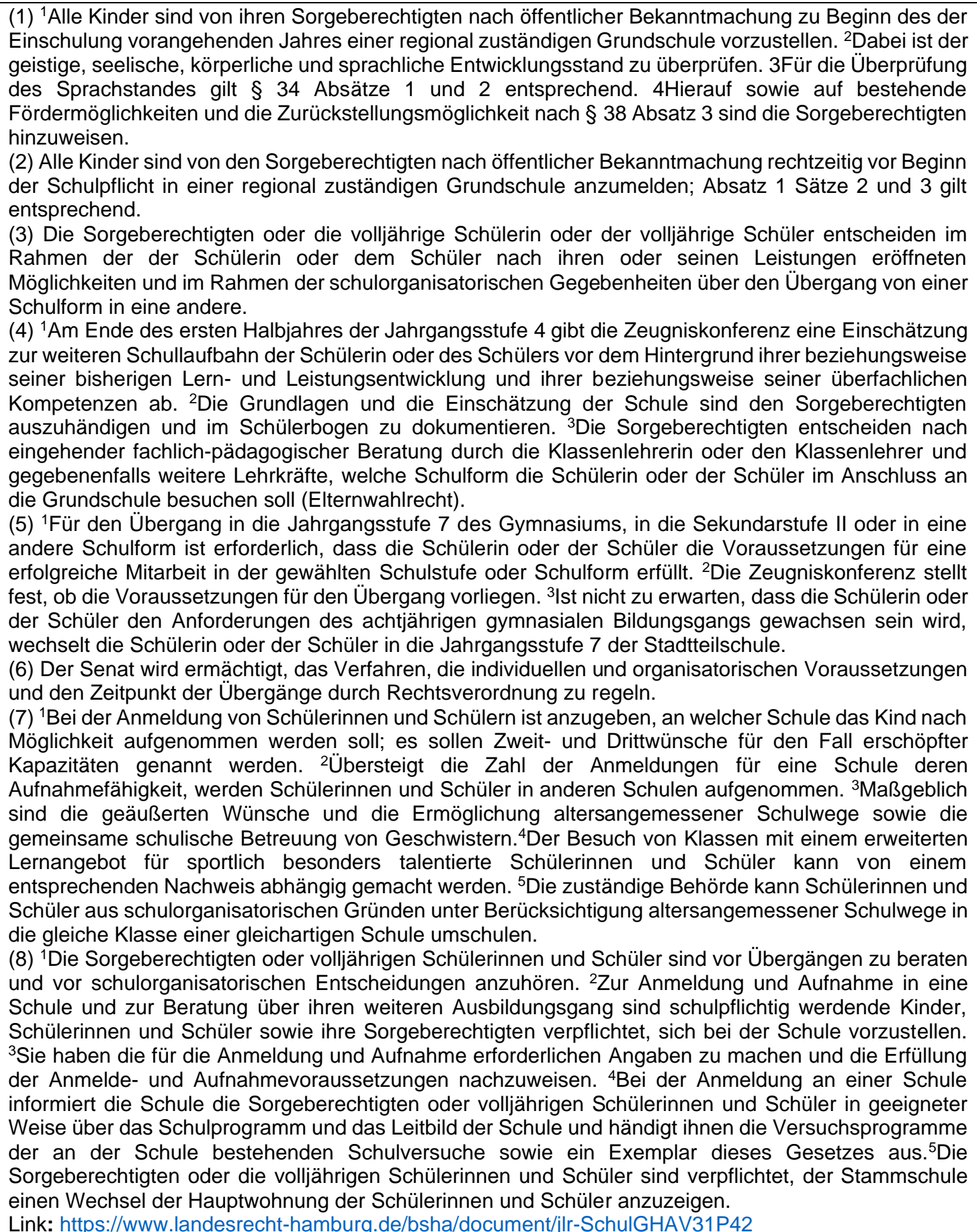 \\
\hline
\end{tabular}

Hessisches Schulgesetz (HSchG)

\begin{tabular}{|l|l|}
\hline Norm & Text \\
\hline §5 & $\begin{array}{l}\text { (1) Alle schulpflichtigen Kinder werden in die allgemeine Schule aufgenommen. Bei Anspruch auf } \\
\text { sonderpädagogische Förderung kann bei der Anmeldung nach § } 58 \text { Abs. 1 Satz } 2 \text { durch die Eltern die } \\
\text { unmittelbare Aufnahme in der Förderschule beantragt werden. Wenn zum Zeitpunkt der Anmeldung } \\
\text { unter Berücksichtigung des bisherigen Bildungsverlaufs des Kindes Anspruch auf sonderpädagogische } \\
\text { Förderung bestehen kann und keine unmittelbare Aufnahme in die Förderschule beantragt wurde, } \\
\text { entscheidet im Rahmen der Abs. } 2 \text { bis } 4 \text { die Schulleiterin oder der Schulleiter nach Anhörung der Eltern } \\
\text { und im Benehmen mit der Schulaufsichtsbehörde über Art, Umfang und Organisation der } \\
\text { sonderpädagogischen Förderung. Auf Antrag der Eltern ist das Verfahren nach Abs. } 2 \text { bis } 4 \text { unmittelbar } \\
\text { nach der Anmeldung durchzuführen. } \\
\text { (2) Kommt ein Anspruch auf sonderpädagogische Förderung bei einer Schülerin oder einem Schüler in } \\
\text { Betracht und reichen allgemeine Maßnahmen der Prävention und der Förderung nicht aus oder sind } \\
\text { solche nicht möglich, wird unverzüglich ein Förderausschuss nach Abs. 3 einberufen. Auf der Grundlage }\end{array}$ \\
\hline
\end{tabular}




\begin{tabular}{|c|c|}
\hline & 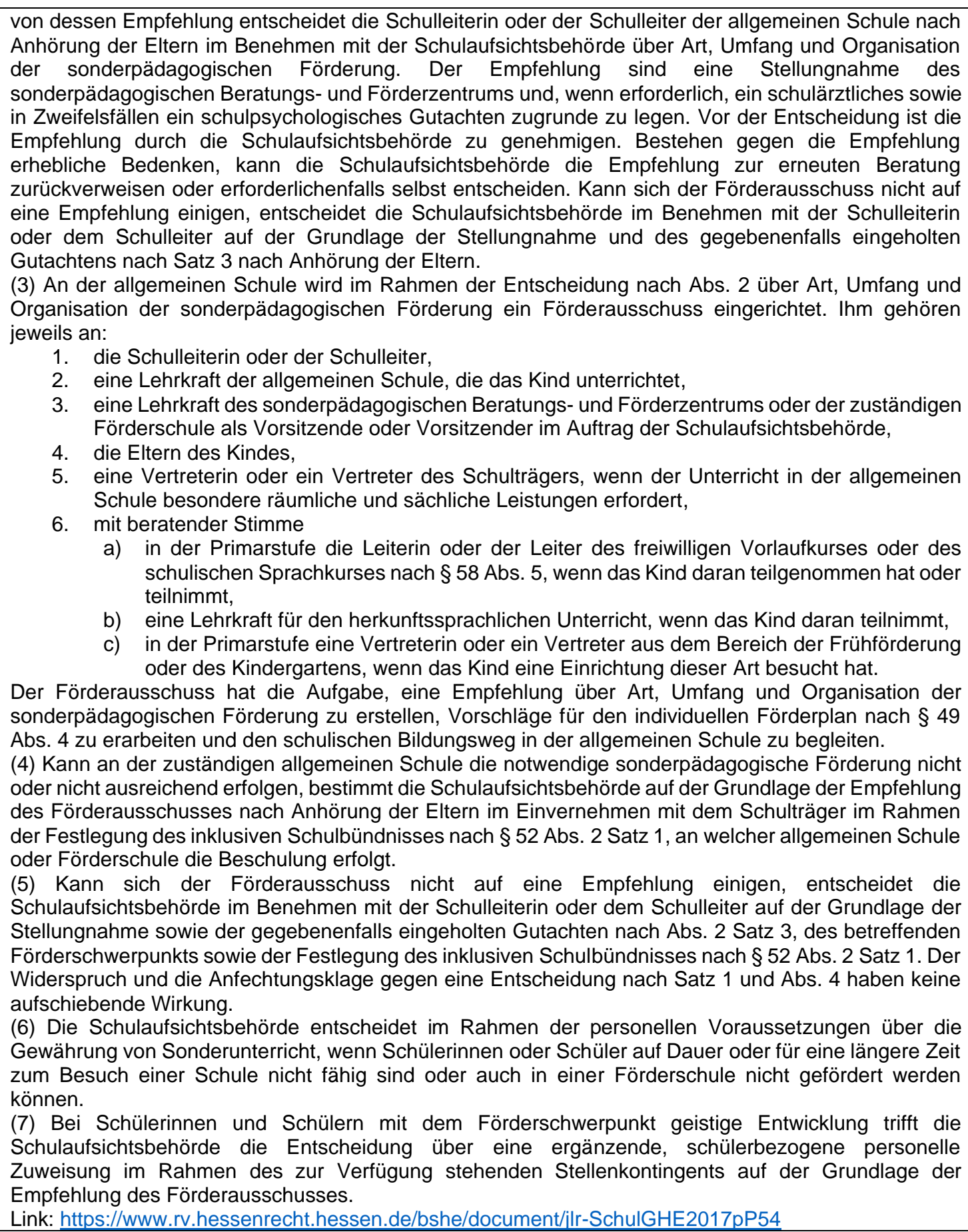 \\
\hline $\begin{array}{l}\S 75 \\
\text { Abs. } 3\end{array}$ & $\begin{array}{l}\text { (3) Schülerinnen und Schüler, die die fünfte oder sechste Jahrgangsstufe der Realschule, des } \\
\text { Gymnasiums oder der entsprechenden Schulzweige schulformbezogener Gesamtschulen besuchen, } \\
\text { können nach Anhörung der Eltern ausnahmsweise am Ende des Schuljahres in eine andere Schulform } \\
\text { versetzt werden (Querversetzung), wenn eine erfolgreiche Mitarbeit im Unterricht des gewählten } \\
\text { Bildungsganges nicht zu erwarten ist und die Wiederholung der Jahrgangsstufe in der besuchten } \\
\text { Schulform oder in dem entsprechenden Zweig der schulformbezogenen Gesamtschule die Schülerin } \\
\text { oder den Schüler in der Entwicklung erheblich beeinträchtigen würde. Die Entscheidung der } \\
\text { Klassenkonferenz bedarf der Zustimmung der Schulleiterin oder des Schulleiters. Der Widerspruch und } \\
\text { die Anfechtungsklage gegen eine Versetzungsentscheidung nach dieser Bestimmung haben keine } \\
\text { aufschiebende Wirkung. Im Schuljahr 2020/2021 sind Satz } 1 \text { bis } 3 \text { auch in der siebenten Jahrgangsstufe } \\
\text { anzuwenden. } \\
\text { Link: https://www.rv.hessenrecht.hessen.de/bshe/document/ilr-SchulGHE2017pG19 }\end{array}$ \\
\hline$\S 77$ & $\begin{array}{l}\text { (1) Die Wahl des Bildungsganges nach dem Besuch der Grundschule ist Sache der Eltern. Wird der } \\
\text { Bildungsgang sowohl schulformbezogen als auch integriert angeboten, können die Eltern zwischen } \\
\text { beiden Formen wählen. Der Besuch eines weiterführenden Bildungsganges setzt Eignung voraus. }\end{array}$ \\
\hline
\end{tabular}




\begin{tabular}{|c|c|}
\hline & $\begin{array}{l}\text { (2) Die Eignung einer Schülerin oder eines Schülers für einen weiterführenden Bildungsgang ist } \\
\text { gegeben, wenn bisherige Lernentwicklung, Leistungsstand und Arbeitshaltung eine erfolgreiche } \\
\text { Teilnahme am Unterricht des gewählten Bildungsganges erwarten lassen. } \\
\text { (3) Bei der Wahl des weiterführenden Bildungsganges haben die Eltern Anspruch auf eingehende } \\
\text { Beratung. Sie teilen inre Entscheidung der Klassenlehrerin oder dem Klassenlehrer der abgebenden } \\
\text { Jahrgangsstufe mit. Zur Entscheidung nimmt die Klassenkonferenz unter dem Vorsitz der Schulleiterin } \\
\text { oder des Schulleiters schriftlich Stellung. Die Stellungnahme muss eine Empfehlung für den } \\
\text { Bildungsgang oder die Bildungsgänge enthalten, für den oder für die die Eignung der Schülerin oder des } \\
\text { Schülers nach Maßgabe des Abs. } 2 \text { gegeben ist. Wird dabei dem Wunsch der Eltern widersprochen, so } \\
\text { ist innen eine erneute Beratung anzubieten. Halten die Eltern ihre Entscheidung aufrecht, so erfolgt die } \\
\text { Aufnahme in den gewählten Bildungsgang. Satz } 4 \text { gilt auch bei der Wahl einer Förderstufe, } \\
\text { Mittelstufenschule oder einer schulformübergreifenden (integrierten) Gesamtschule. } \\
\text { (4) Ist bei der Wahl einer Förderstufe oder einer schulformübergreifenden (integrierten) Gesamtschule } \\
\text { die Aufnahme in eine Förderstufe oder in eine schulformübergreifende (integrierte) Gesamtschule nicht } \\
\text { möglich, gilt für den Übergang in einen weiterführenden Bildungsgang Abs. } 3 \text { Satz } 2 \text { bis } 6 \text { entsprechend. } \\
\text { (5) An schulformübergreifenden (integrierten) Gesamtschulen (\$ } 27 \text { ) sind die Informations- und } \\
\text { Entscheidungsrechte der Eltern bei der Ersteinstufung von Schülerinnen und Schülern in } \\
\text { Fachleistungskurse den Vorschriften des Abs. } 3 \text { entsprechend zu wahren. } \\
\text { (6) Für die endgültige Entscheidung über den weiteren Bildungsweg am Ende der Förderstufe gilt Abs. } \\
3 \text { Satz } 1 \text { bis } 5 \text { entsprechend. Der Übergang in den Bildungsgang der Realschule oder des Gymnasiums } \\
\text { setzt voraus, dass ihn die Klassenkonferenz der abgebenden Förderstufe befürwortet. } \\
\text { Link: https://www.rv.hessenrecht.hessen.de/bshe/document/Ilr-SchulGHE2017pP77 }\end{array}$ \\
\hline $\begin{array}{l}\S 182 \\
\text { Abs. } 1\end{array}$ & $\begin{array}{l}\text { (1) Wer einen anderen der Schulpflicht dauernd oder hartnäckig wiederholt entzieht, wird mit } \\
\text { Freiheitsstrafe bis zu sechs Monaten oder mit Geldstrafe bis zu einhundertachtzig Tagessätzen bestraft. }\end{array}$ \\
\hline
\end{tabular}

Infektionsschutzgesetz (IfSG)

\begin{tabular}{|c|c|}
\hline Norm & ext \\
\hline$\S 28$ & $\begin{array}{l}\text { (1) Werden Kranke, Krankheitsverdächtige, Ansteckungsverdächtige oder Ausscheider festgestellt oder } \\
\text { ergibt sich, dass ein Verstorbener krank, krankheitsverdächtig oder Ausscheider war, so trifft die } \\
\text { zuständige Behörde die notwendigen Schutzmaßnahmen, insbesondere die in } \S 28 a \text { Absatz } 1 \text { und in } \\
\text { den } \S \S 29 \text { bis } 31 \text { genannten, soweit und solange es zur Verhinderung der Verbreitung übertragbarer } \\
\text { Krankheiten erforderlich ist; sie kann insbesondere Personen verpflichten, den Ort, an dem sie sich } \\
\text { befinden, nicht oder nur unter bestimmten Bedingungen zu verlassen oder von ihr bestimmte Orte oder } \\
\text { öffentliche Orte nicht oder nur unter bestimmten Bedingungen zu betreten. Unter den Voraussetzungen } \\
\text { von Satz } 1 \text { kann die zuständige Behörde Veranstaltungen oder sonstige Ansammlungen von Menschen } \\
\text { beschränken oder verbieten und Badeanstalten oder in } \S 33 \text { genannte Gemeinschaftseinrichtungen oder } \\
\text { Teile davon schließen. Eine Heilbehandlung darf nicht angeordnet werden. Die Grundrechte der } \\
\text { körperlichen Unversehrtheit (Artikel } 2 \text { Absatz } 2 \text { Satz } 1 \text { des Grundgesetzes), der Freiheit der Person } \\
\text { (Artikel } 2 \text { Absatz } 2 \text { Satz } 2 \text { des Grundgesetzes), der Versammlungsfreiheit (Artikel } 8 \text { des Grundgesetzes), } \\
\text { der Freizügigkeit (Artikel } 11 \text { Absatz } 1 \text { des Grundgesetzes) und der Unverletzlichkeit der Wohnung (Artikel } \\
13 \text { Absatz } 1 \text { des Grundgesetzes) werden insoweit eingeschränkt. } \\
\text { (2) Wird festgestellt, dass eine Person in einer Gemeinschaftseinrichtung an Masern erkrankt, dessen } \\
\text { verdächtig oder ansteckungsverdächtig ist, kann die zuständige Behörde Personen, die weder einen } \\
\text { Impfschutz, der den Empfehlungen der Ständigen Impfkommission entspricht, noch eine Immunität } \\
\text { gegen Masern durch ärztliches Zeugnis nachweisen können, die in } \S 34 \text { Absatz } 1 \text { Satz } 1 \text { und } 2 \\
\text { genannten Verbote erteilen, bis eine Weiterverbreitung der Krankheit in der Gemeinschaftseinrichtung } \\
\text { nicht mehr zu befürchten ist. } \\
\text { (3) Für Maßnahmen nach den Absätzen } 1 \text { und } 2 \text { gilt } § 16 \text { Abs. } 5 \text { bis } 8 \text {, für ihre Überwachung außerdem } \\
\S 16 \text { Abs. } 2 \text { entsprechend. } \\
\text { Link: http://www.gesetze-im-internet.de/ifsg/ } 28 \text {.html }\end{array}$ \\
\hline$\S 28 a$ & $\begin{array}{l}\text { (1) Notwendige Schutzmaßnahmen im Sinne des } \S 28 \text { Absatz } 1 \text { Satz } 1 \text { und } 2 \text { zur Verhinderung der } \\
\text { Verbreitung der Coronavirus-Krankheit-2019 (COVID-19) können für die Dauer der Feststellung einer } \\
\text { epidemischen Lage von nationaler Tragweite nach § } 5 \text { Absatz } 1 \text { Satz } 1 \text { durch den Deutschen Bundestag } \\
\text { insbesondere sein } \\
\text { 1. Anordnung eines Abstandsgebots im öffentlichen Raum, } \\
\text { 2. Verpflichtung zum Tragen einer Mund-Nasen-Bedeckung (Maskenpflicht), } \\
\text { 2a. Verpflichtung zur Vorlage eines Impf-, Genesenen- oder Testnachweises. } \\
\text { 3. Ausgangs- oder Kontaktbeschränkungen im privaten sowie im öffentlichen Raum, } \\
\text { 4. Verpflichtung zur Erstellung und Anwendung von Hygienekonzepten für Betriebe, } \\
\text { Einrichtungen oder Angebote mit Publikumsverkehr, } \\
\text { 5. Untersagung oder Beschränkung von Freizeitveranstaltungen und ähnlichen Veranstaltungen, } \\
\text { 6. Untersagung oder Beschränkung des Betriebs von Einrichtungen, die der Freizeitgestaltung } \\
\text { 7. Unzurechnen sind, } \\
\text { 8. Kultureinrichtungen, } \\
\text { Untersagung oder Beschränkung von Sportveranstaltungen und der Sportausübung, }\end{array}$ \\
\hline
\end{tabular}


9. umfassendes oder auf bestimmte Zeiten beschränktes Verbot der Alkoholabgabe oder des Alkoholkonsums auf bestimmten öffentlichen Plätzen oder in bestimmten öffentlich zugänglichen Einrichtungen,

10. Untersagung von oder Erteilung von Auflagen für das Abhalten von Veranstaltungen, Ansammlungen, Aufzügen, Versammlungen sowie religiösen oder weltanschaulichen Zusammenkünften,

11. Untersagung oder Beschränkung von Reisen; dies gilt insbesondere für touristische Reisen,

12. Untersagung oder Beschränkung von Übernachtungsangeboten,

13. Untersagung oder Beschränkung des Betriebs von gastronomischen Einrichtungen,

14. Schließung oder Beschränkung von Betrieben, Gewerben, Einzel- oder Großhandel,

15. Untersagung oder Beschränkung des Betretens oder des Besuchs von Einrichtungen des Gesundheits- oder Sozialwesens,

16. Schließung von Gemeinschaftseinrichtungen im Sinne von $\S 33$, Hochschulen, außerschulischen Einrichtungen der Erwachsenenbildung oder ähnlichen Einrichtungen oder Erteilung von Auflagen für die Fortführung ihres Betriebs oder

17. Anordnung der Verarbeitung der Kontaktdaten von Kunden, Gästen oder Veranstaltungsteilnehmern, um nach Auftreten einer Infektion mit dem Coronavirus SARSCoV-2 mögliche Infektionsketten nachverfolgen und unterbrechen zu können.

(2) Die Anordnung der folgenden Schutzmaßnahmen nach Absatz 1 in Verbindung mit $\S 28$ Absatz 1 ist nur zulässig, soweit auch bei Berücksichtigung aller bisher getroffenen anderen Schutzmaßnahmen eine wirksame Eindämmung der Verbreitung der Coronavirus-Krankheit-2019 (COVID-19) erheblich gefährdet wäre:

1. Untersagung von Versammlungen oder Aufzügen im Sinne von Artikel 8 des Grundgesetzes und von religiösen oder weltanschaulichen Zusammenkünften nach Absatz 1 Nummer 10,

2. Anordnung einer Ausgangsbeschränkung nach Absatz 1 Nummer 3, nach der das Verlassen des privaten Wohnbereichs nur zu bestimmten Zeiten oder zu bestimmten Zwecken zulässig ist, und

3. Untersagung des Betretens oder des Besuchs von Einrichtungen im Sinne von Absatz 1 Nummer 15, wie zum Beispiel Alten- oder Pflegeheimen, Einrichtungen der Behindertenhilfe, Entbindungseinrichtungen oder Krankenhäusern für enge Angehörige von dort behandelten, gepflegten oder betreuten Personen.

Schutzmaßnahmen nach Absatz 1 Nummer 15 dürfen nicht zur vollständigen Isolation von einzelnen Personen oder Gruppen führen; ein Mindestmaß an sozialen Kontakten muss gewährleistet bleiben.

(3) Entscheidungen über Schutzmaßnahmen zur Verhinderung der Verbreitung der CoronavirusKrankheit-2019 (COVID-19) nach Absatz 1 in Verbindung mit $§ 28$ Absatz 1, nach § 28 Absatz 1 Satz 1 und 2 und den $\S \S 29$ bis 32 sind insbesondere an dem Schutz von Leben und Gesundheit und der Funktionsfähigkeit des Gesundheitssystems auszurichten; dabei sind absehbare Änderungen des Infektionsgeschehens durch ansteckendere, das Gesundheitssystem stärker belastende Virusvarianten zu berücksichtigen. Zum präventiven Infektionsschutz können insbesondere die in Absatz 1 Nummer 1, 2, 2a, 4 und 17 genannten Schutzmaßnahmen ergriffen werden. Weitergehende Schutzmaßnahmen sollen unter Berücksichtigung des jeweiligen regionalen und überregionalen Infektionsgeschehens mit dem Ziel getroffen werden, eine drohende Überlastung der regionalen und überregionalen stationären Versorgung zu vermeiden. Wesentlicher Maßstab für die weitergehenden Schutzmaßnahmen ist insbesondere die Anzahl der in Bezug auf die Coronavirus-Krankheit-2019 (COVID-19) in ein Krankenhaus aufgenommenen Personen je 100000 Einwohner innerhalb von sieben Tagen. Weitere Indikatoren wie die unter infektionsepidemiologischen Aspekten differenzierte Anzahl der Neuinfektionen mit dem Coronavirus SARS-CoV-2 je 100000 Einwohner innerhalb von sieben Tagen, die verfügbaren intensivmedizinischen Behandlungskapazitäten und die Anzahl der gegen die Coronavirus-Krankheit2019 (COVID-19) geimpften Personen sollen bei der Bewertung des Infektionsgeschehens berücksichtigt werden. Die Landesregierungen können im Rahmen der Festlegung der Schutzmaßnahmen unter Berücksichtigung der jeweiligen stationären Versorgungskapazitäten in einer Rechtsverordnung nach $\S 32$ Schwellenwerte für die Indikatoren nach den Sätzen 4 und 5 festsetzen; entsprechend können die Schutzmaßnahmen innerhalb eines Landes regional differenziert werden. Das Robert Koch-Institut veröffentlicht im Internet unter https://www.rki.de/covid-19-trends werktäglich nach Altersgruppen differenzierte und mindestens auf einzelne Länder und auf das Bundesgebiet bezogene Daten zu Indikatoren nach den Sätzen 4 und 5. Die Länder können die Indikatoren nach den Sätzen 4 und 5 landesweit oder regional differenziert auch statt bezogen auf 100000 Einwohner bezogen auf das Land oder die jeweilige Region als Maßstab verwenden.

(4) Im Rahmen der Kontaktdatenerhebung nach Absatz 1 Nummer 17 dürfen von den Verantwortlichen nur personenbezogene Angaben sowie Angaben zum Zeitraum und zum Ort des Aufenthaltes erhoben und verarbeitet werden, soweit dies zur Nachverfolgung von Kontaktpersonen zwingend notwendig ist. Die Verantwortlichen haben sicherzustellen, dass eine Kenntnisnahme der erfassten Daten durch Unbefugte ausgeschlossen ist. Die Daten dürfen nicht zu einem anderen Zweck als der Aushändigung auf Anforderung an die nach Landesrecht für die Erhebung der Daten zuständigen Stellen verwendet werden und sind vier Wochen nach Erhebung zu löschen. Die zuständigen Stellen nach Satz 3 sind berechtigt, die erhobenen Daten anzufordern, soweit dies zur Kontaktnachverfolgung nach § 25 Absatz 1 erforderlich ist. Die Verantwortlichen nach Satz 1 sind in diesen Fällen verpflichtet, den zuständigen Stellen nach Satz 3 die erhobenen Daten zu übermitteln. Eine Weitergabe der übermittelten Daten durch 


\begin{tabular}{|c|c|}
\hline & 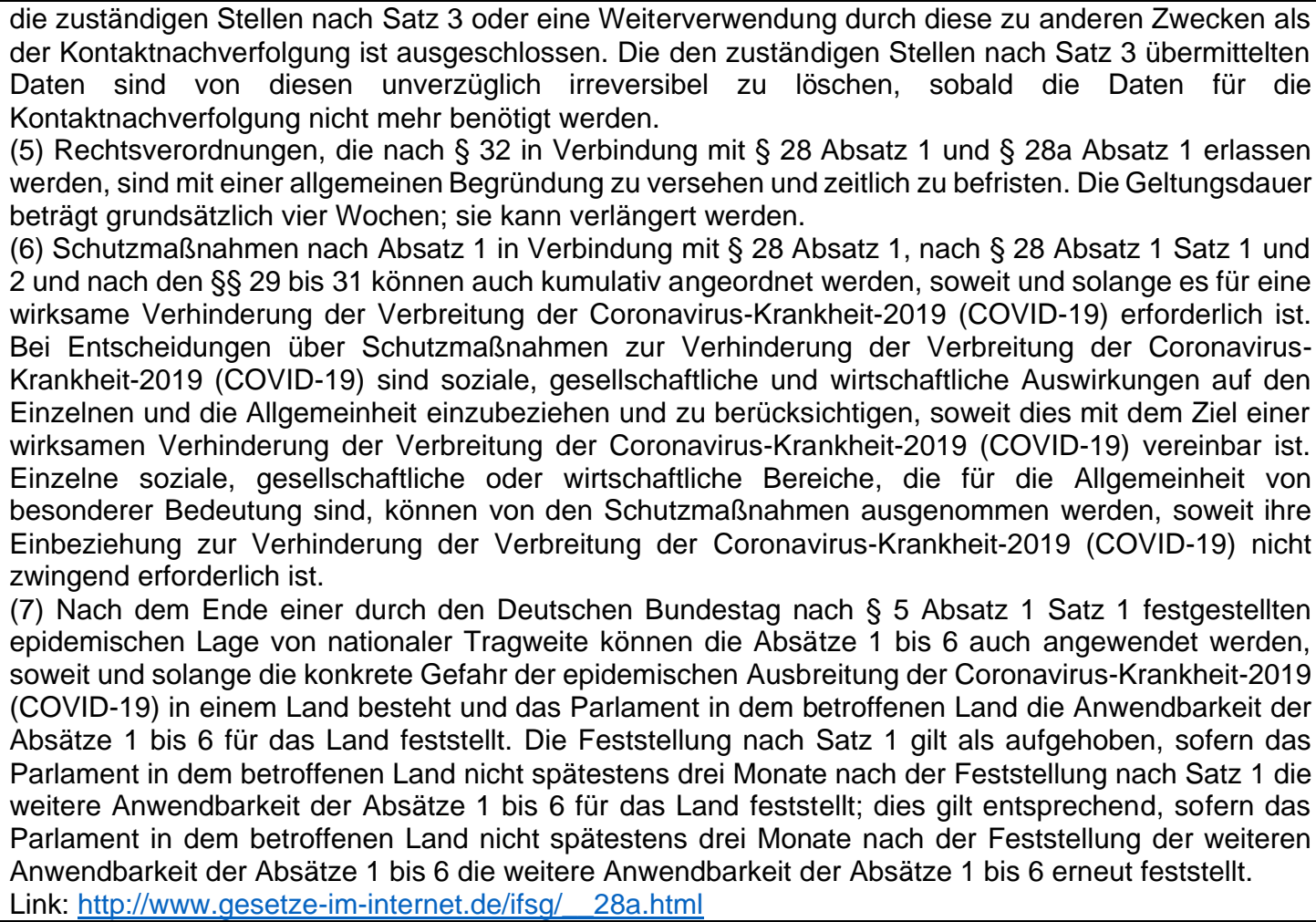 \\
\hline$\S 3$ & $\begin{array}{l}\text { Die Landesregierungen werden ermächtigt, unter den Voraussetzungen, die für Maßnahmen nach den } \\
\S \S 28,28 \text { a und } 29 \text { bis } 31 \text { maßgebend sind, auch durch Rechtsverordnungen entsprechende Gebote und } \\
\text { Verbote zur Bekämpfung übertragbarer Krankheiten zu erlassen. Die Landesregierungen können die } \\
\text { Ermächtigung durch Rechtsverordnung auf andere Stellen übertragen. Die Grundrechte der körperlichen } \\
\text { Unversehrtheit (Artikel } 2 \text { Absatz } 2 \text { Satz } 1 \text { des Grundgesetzes), der Freiheit der Person (Artikel } 2 \text { Absatz } \\
2 \text { Satz } 2 \text { des Grundgesetzes), der Freizügigkeit (Artikel } 11 \text { Absatz } 1 \text { des Grundgesetzes), der } \\
\text { Versammlungsfreiheit (Artikel } 8 \text { des Grundgesetzes), der Unverletzlichkeit der Wohnung (Artikel } 13 \\
\text { Absatz } 1 \text { des Grundgesetzes) und des Brief- und Postgeheimnisses (Artikel } 10 \text { des Grundgesetzes) } \\
\text { können insoweit eingeschränkt werden. } \\
\text { Link: http://www.gesetze-im-internet.de/ifsg/ } 32 . h t m l\end{array}$ \\
\hline
\end{tabular}

Niedersächsisches Schulgesetz (NSchG)

\begin{tabular}{|c|c|}
\hline Norm & ext \\
\hline $\begin{array}{l}\text { § } 4 \\
\text { Abs. } 1 \\
\text { Satz } 2\end{array}$ & $\begin{array}{l}\text { (1) [...] }{ }^{2} \text { Welche Schulform die Schülerinnen und Schüler besuchen, entscheiden die } \\
\text { Erziehungsberechtigten }(\$ 59 \text { Abs. } 1 \text { Satz } 1) . \\
\text { Link: } \text { http://www.schure.de/2241001/nschg.htm\#p4 }\end{array}$ \\
\hline $\begin{array}{l}\S 6 \\
\text { Abs. } 5\end{array}$ & $\begin{array}{l}\text { (5) 1Die Grundschule bietet im 4. Schuljahrgang den Erziehungsberechtigten mindestens zwei } \\
\text { Gespräche an, um sie über die individuelle Lernentwicklung ihres Kindes zu informieren und über die } \\
\text { Wahl der weiterführenden Schulform zu beraten. 2Die Erziehungsberechtigten entscheiden in eigener } \\
\text { Verantwortung über die Schulform ihrer Kinder (\$ } 59 \mathrm{Abs.1} \text { Satz 1). } \\
\text { Link: http://www.schure.de/2241001/nschg.htm\#p6 }\end{array}$ \\
\hline $\begin{array}{l}\S 59 \\
\text { Abs. } 5 \\
\text { Satz } 1\end{array}$ & $\begin{array}{l}\text { (5) 1Eine Schülerin oder ein Schüler kann auf Vorschlag der Schule durch die Schulbehörde an die } \\
\text { Schule einer anderen, für sie oder ihn geeigneten Schulform überwiesen werden, wenn sie oder er auch } \\
\text { unter Beachtung der Anforderungen an eine inklusive Schule }(\$ 4) \text { nur an der anderen Schule } \\
\text { hinreichend gefördert werden kann und ihr oder sein Kindeswohl den Schulwechsel erfordert; die } \\
\text { Schulbehörde hat in regelmäßigen Abständen zu überprüfen, ob die Voraussetzungen für die } \\
\text { Überweisung weiterhin vorliegen. } \\
\text { Link: http://www.schure.de/2241001/nschg.htm\#p59 }\end{array}$ \\
\hline
\end{tabular}

Sächsisches Schulgesetz (SächsSchG) und Schulordnung Gymnasien Abiturprüfung (SächsSOGYA)

\begin{tabular}{|l|l|}
\hline Norm & Text \\
\hline 34 & $\begin{array}{l}\text { (1) 1 Über den Wechsel von der Grundschule auf eine weiterführende allgemeinbildende Schule } \\
\text { entscheiden die Eltern auf Empfehlung der Schule. 2Die Grundschule berät die Eltern über die für den } \\
\text { Schüler geeignete Schulart und gibt in der Klassenstufe } 4 \text { eine schriftliche Bildungsempfehlung. }{ }^{3} \text { Die }\end{array}$ \\
& $\begin{array}{l}\text { Bildungsempfehlung für das Gymnasium wird erteilt, wenn } \\
\text { 1. } \quad \text { der Durchschnitt der Noten in den Fächern Deutsch, Mathematik und Sachunterricht in der } \\
\text { Halbjahresinformation oder am Ende des Schuljahres 2,0 oder besser ist und keines dieser } \\
\text { Fächer mit der Note „ausreichend“ oder schlechter benotet wurde und }\end{array}$ \\
\hline
\end{tabular}




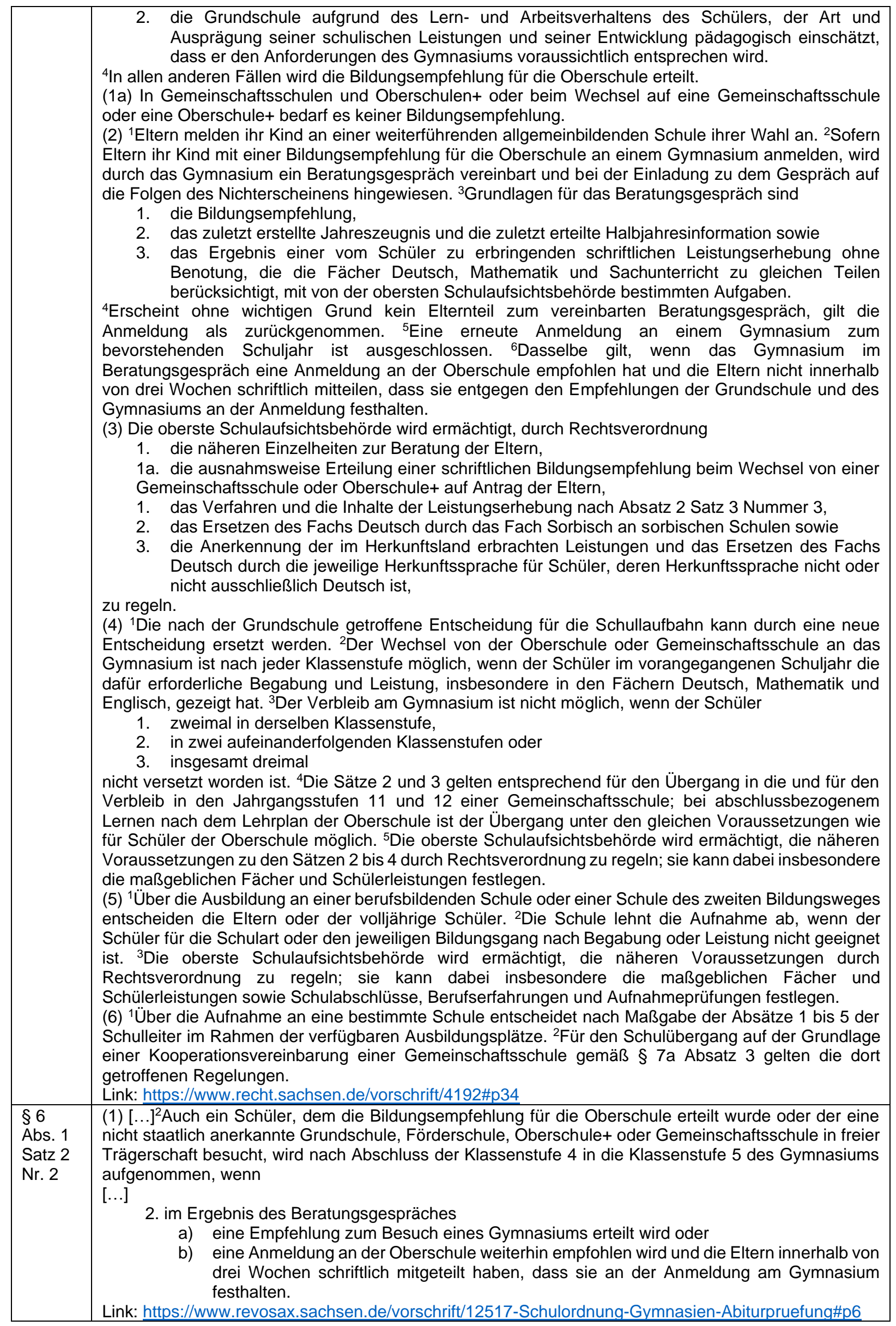




\begin{tabular}{|c|c|}
\hline Norm & Text \\
\hline $\begin{array}{l}\text { § } 9 \\
\text { Abs. } 3\end{array}$ & $\begin{array}{l}\text { (3) An den Gymnasien bilden die ersten beiden Jahrgangsstufen die Orientierungsstufe. Die } \\
\text { Orientierungsstufe dient der Erprobung, der Förderung und der Beobachtung der Schülerin oder des } \\
\text { Schülers, um in Zusammenarbeit mit den Eltern die Entscheidung über die Eignung der Schülerin oder } \\
\text { des Schülers für das Gymnasium abzusichern. Das Gymnasium hat seinen Unterricht so zu gestalten } \\
\text { und die Schülerin oder den Schüler so zu fördern, dass die Versetzung in die Jahrgangsstufe } 7 \text { am } \\
\text { Gymnasium der Regelfall ist. Das Gymnasium weist die Schülerin oder den Schüler mit dem Abschluss } \\
\text { der Orientierungsstufe der nächsten Jahrgangsstufe der Gemeinschaftsschule nur zu } \\
\text { (Schrägversetzung), wenn die Leistungen trotz der individuellen Förderung den Anforderungen des } \\
\text { Gymnasiums nicht genügen. } \\
\text { Link: } \\
\text { rechtsprechung.sh.juris.de/jportal/portal/t/r7s/page/bsshoprod.psml?pid=Dokumentanzeige\&showdocc } \\
\text { ase=1\&js peid=Trefferliste\&documentnumber=1\&numberofresults=1\&fromdoctodoc=yes\&doc.id=ilr- }\end{array}$ \\
\hline$\S 24$ & 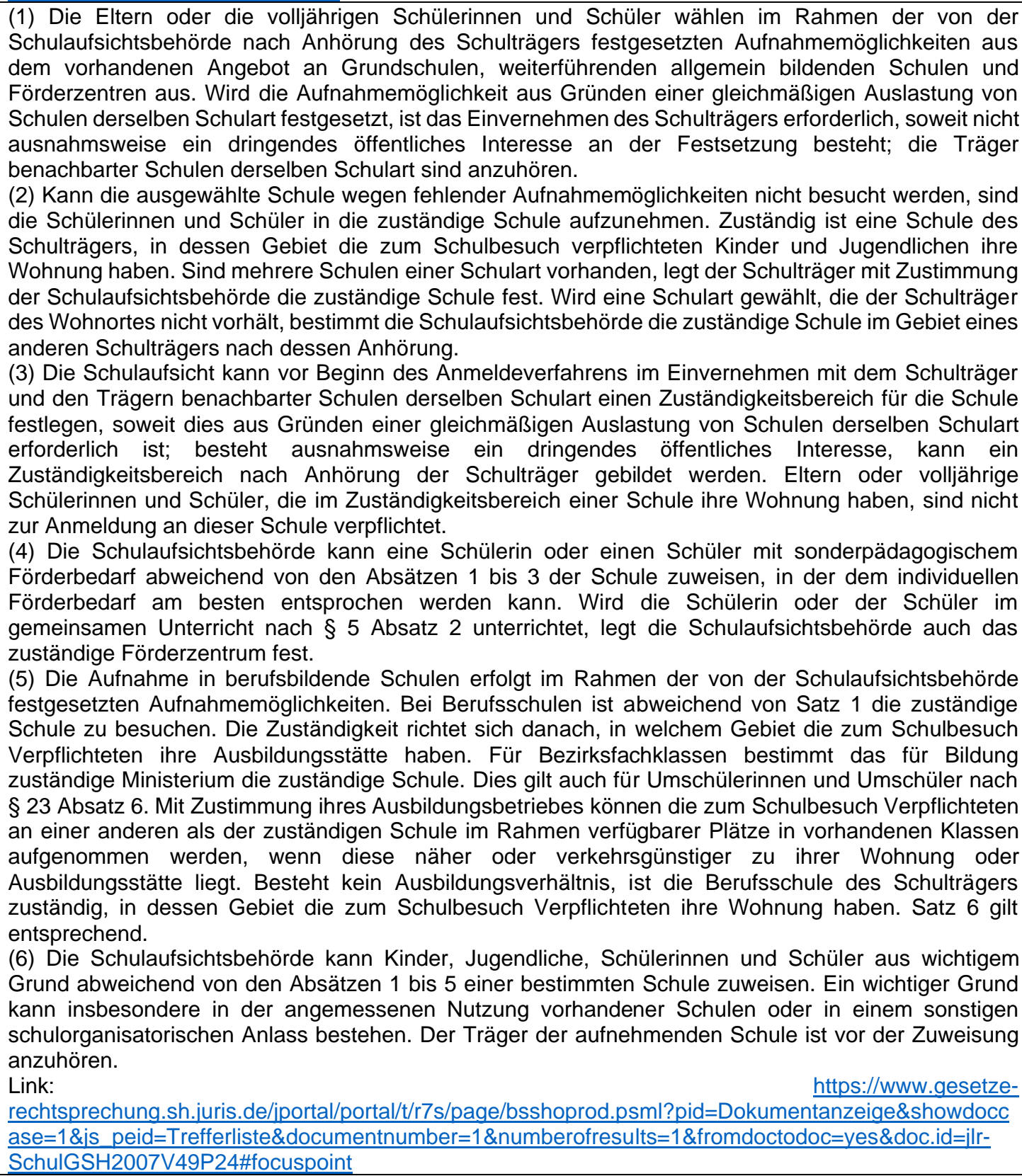 \\
\hline
\end{tabular}

Schulgesetz für Baden-Württemberg (SchG B-W) und Aufnahmeverordnung (AufnV BW)

\begin{tabular}{|l|l|}
\hline Norm & Text \\
\hline § 5 & (2) Die Grundschule berät die Erziehungsberechtigten, welche der auf ihr aufbauenden Schularten für \\
Abs.2 & das Kind geeignet ist. Hierbei werden neben dem Leistungsstand auch die soziale und psychische Reife \\
SchG & $\begin{array}{l}\text { sowie das Entwicklungspotenzial des Kindes betrachtet. Es wird über die möglichen Angebote aufgeklärt } \\
\text { und die Auswirkungen der Entscheidung der Erziehungsberechtigten werden dargelegt. Die }\end{array}$ \\
\hline
\end{tabular}

DDS, 113. Jg., 4(2021) 14 


\begin{tabular}{|c|c|}
\hline & $\begin{array}{l}\text { Grundschule erteilt eine Empfehlung, welche weiterführende Schulart das Kind aus pädagogisch- } \\
\text { fachlicher Sicht besuchen soll (Grundschulempfehlung). Die Erziehungsberechtigten legen als Teil der } \\
\text { Anmeldung die Grundschulempfehlung der weiterführenden Schule vor. Die freie Entscheidung der } \\
\text { Erziehungsberechtigten für eine der auf der Grundschule aufbauenden Schularten bleibt hiervon } \\
\text { unberührt. } \\
\text { Link: } \\
\text { bw.de/portal/portal///csq/page/bsbawueprod.psml?pid=Dokumentanzeige\&showdoccase=1\&js peid=T } \\
\text { refferliste\&documentnumber=1\&numberofresults=1\&fromdoctodoc=yes\&doc.id=|lr- } \\
\text { SchulGBW1983V44P5\#focuspoint }\end{array}$ \\
\hline $\begin{array}{l}\text { §3 } \\
\text { AufnV }\end{array}$ & $\begin{array}{l}\text { Die Erziehungsberechtigten entscheiden, welche weiterführende Schulart ihr Kind besucht. Sie legen } \\
\text { die Grundschulempfehlung nach } \S 5 \text { Absatz } 2 \text { Satz } 5 \text { SchG der aufnehmenden Schule bei der } \\
\text { Anmeldung vor. } \\
\text { Link: } \\
\text { bw.de/iportal/portal/t/ci3/page/bsbawueprod.psml?pid=Dokumentanzeige\&showdoccase }=1 \& \text { is peid=Tr } \\
\text { efferliste\&documentnumber=1\&numberofresults=1\&fromdoctodoc=yes\&doc.id=jlr- } \\
\text { AufnVBW2011V1P3\#focuspoint }\end{array}$ \\
\hline
\end{tabular}

Schulgesetz für das Land Berlin

\begin{tabular}{|c|c|}
\hline Norm & Text \\
\hline$\S 56$ & 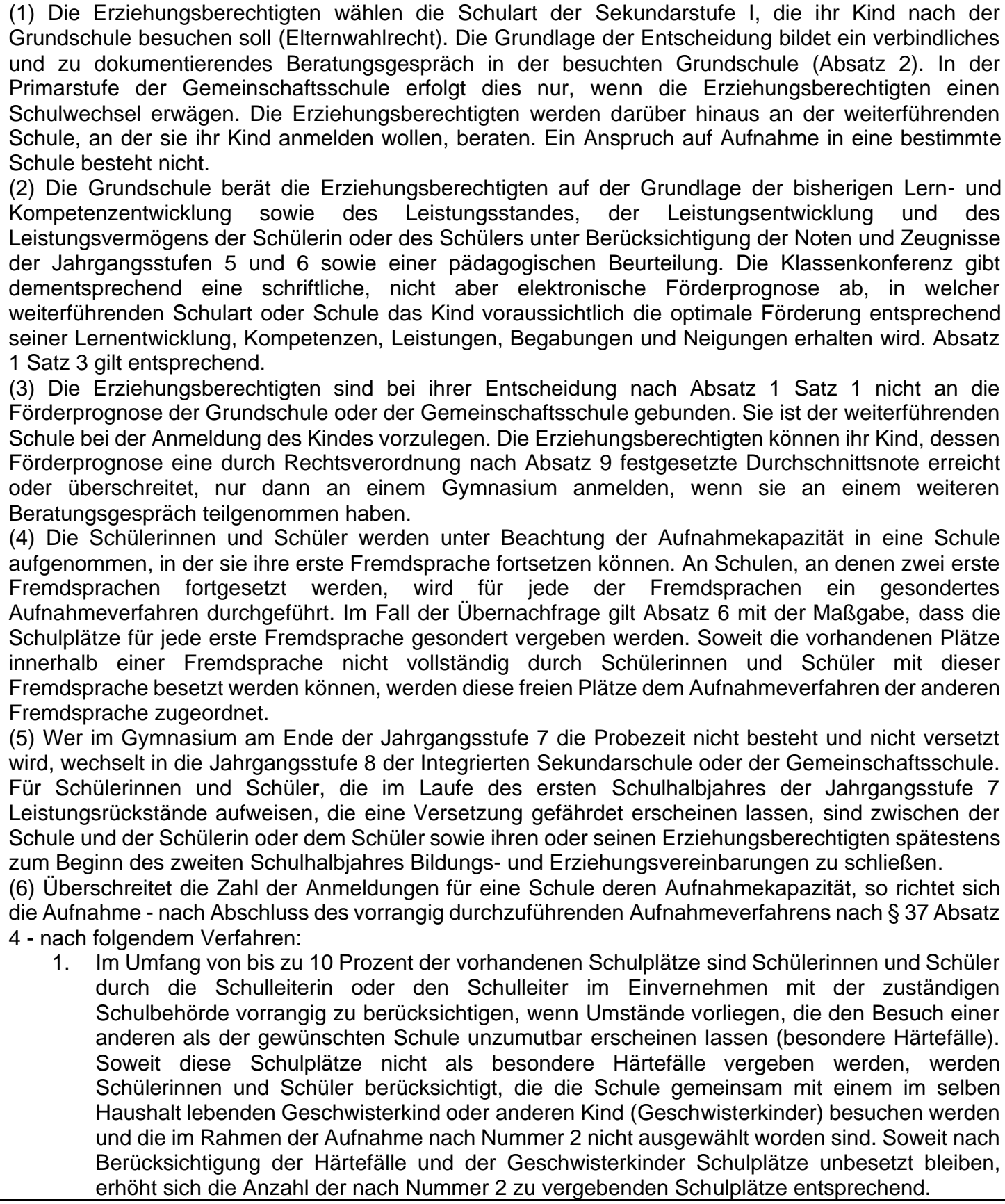 \\
\hline
\end{tabular}


2. Mindestens 60 Prozent der Schulplätze werden nach Aufnahmekriterien vergeben, die von der Schule unter Berücksichtigung des Schulprogramms festgelegt werden. Zur Feststellung, ob eine Schülerin oder ein Schüler die Aufnahmekriterien der Schule erfüllt, ist ein Verfahren für die Aufnahme durchzuführen. Die Grundlagen der Aufnahmeentscheidung sind zu dokumentieren. Die Aufnahmekriterien und die Gestaltung des Verfahrens für die Aufnahme unterliegen der Genehmigung durch die Schulaufsichtsbehörde, hinsichtlich der Aufnahmekriterien im Benehmen, hinsichtlich der Gestaltung des Verfahrens für die Aufnahme im Einvernehmen mit der jeweils zuständigen Schulbehörde.

3. 30 Prozent der Schulplätze werden durch Los vergeben. Soweit Geschwisterkinder nicht gemäß Nummer 1 oder Nummer 2 berücksichtigt wurden, sind sie vorrangig aufzunehmen. Befinden sich mehrere Geschwisterkinder gleichzeitig ausschließlich im Losverfahren, führt die Aufnahme des ersten Geschwisterkindes durch Los dazu, dass seine weiteren sich im Losverfahren befindenden Geschwister ebenfalls aufgenommen werden.

Das Aufnahmeverfahren nach Satz 1 Nummer 1 bis 3 gilt auch für die Aufnahme in die Jahrgangsstufe 7 der Gemeinschaftsschule mit der Maßgabe, dass in die Jahrgangsstufe 7 zunächst die Schülerinnen und Schüler der eigenen Primarstufe aufrücken. Abweichend von den Nummern 2 und 3 werden an der Gemeinschaftsschule nach Berücksichtigung der Geschwisterkinder alle verbleibenden Schulplätze nach von der Schule festgelegten Aufnahmekriterien vergeben, die eine leistungsheterogene Zusammensetzung der Schülerinnen und Schüler gewährleisten. Schülerinnen und Schüler aller Förderprognosen sind unabhängig von der Durchschnittsnote gleichberechtigt zu berücksichtigen; das Losverfahren kann die Aufnahme nach Kriterien ersetzen.

(7) Kann die Schülerin oder der Schüler nicht gemäß dem Erstwunsch ihrer oder seiner Erziehungsberechtigten in die von ihnen ausgewählte Schule aufgenommen werden, so wird ihren oder seinen Erziehungsberechtigten von der zuständigen Schulbehörde eine aufnahmefähige Schule unter Berücksichtigung der Zweit- und Drittwünsche benannt. Dabei werden Schülerinnen und Schüler vorrangig berücksichtigt, die in dem Bezirk wohnen (§ 41 Absatz 5), in dem die Schule liegt. Kann die Schülerin oder der Schüler auch an dieser Schule nicht aufgenommen werden oder nehmen die Erziehungsberechtigten dieses Angebot nicht wahr, so wird die Schülerin oder der Schüler gemäß $§ 54$ Absatz 3 unter Berücksichtigung der möglichen Kapazitäten einer Schule der gewünschten Schulart zugewiesen. Ist die gewünschte Schulart eine Integrierte Sekundarschule oder eine Gemeinschaftsschule, ist eine Zuweisung in die jeweils andere Schulart zulässig, wenn dies wegen nicht ausreichender Plätze in einer der Schularten erforderlich ist.

(8) Für den Übergang in Jahrgangsstufe 5 gelten die Absätze 1 bis 6 entsprechend, soweit nicht eine auf Grund des Absatzes 9 Satz 1 Nummer 4 oder $\S 18$ Absatz 3 erlassene Rechtsverordnung Abweichendes regelt.

(9) Die für das Schulwesen zuständige Senatsverwaltung wird ermächtigt, das Nähere über den Übergang und die Aufnahme in die Sekundarstufe I durch Rechtsverordnung zu regeln, insbesondere

1. das Verfahren und die Kriterien für die Förderprognose, die Festsetzung der Durchschnittsnote nach Absatz 3 Satz 3 und die verbindlichen Beratungsgespräche gemäß Absatz 2 und 3,

2. die Einzelheiten der Aufnahmekriterien der Schule im Sinne von Absatz 6, wobei als Kriterien insbesondere in Betracht kommen:

a) Leistung und Kompetenzen,

b) Übereinstimmung des Leistungsbildes oder der sonstigen persönlichen Voraussetzungen der Schülerin oder des Schülers mit den Ausprägungen des Schulprogramms,

c) das Ergebnis eines Auswahlgesprächs oder eines anderen spezifischen Eignungsfeststellungsverfahrens;

die Anwendung der Aufnahmekriterien bei der Aufnahmeentscheidung sowie das Nähere über das Verfahren für die Aufnahme einschließlich der Eignungsfeststellung, die Festlegung, ob die Aufnahme unbeschadet von Absatz 6 Nummer 1 zunächst nach Absatz 6 Nummer 2 oder Nummer 3 erfolgt, sowie die Besonderheiten für den Fall, dass es an einer Festlegung der Aufnahmekriterien oder eines Verfahrens für die Aufnahme fehlt,

3. besondere Härtefälle nach Absatz 6 Nummer 1,

4. die Besonderheiten für den altsprachlichen Bildungsgang.

Abweichend von Satz 1 Nummer 2 Buchstabe a und b finden an der Gemeinschaftsschule die Leistung und das Leistungsbild als alleinige Aufnahmekriterien keine Anwendung, das Eignungsfeststellungsverfahren nach Satz 1 Nummer 2 Buchstabe $c$ darf nicht allein auf Leistungskriterien abstellen. In der Rechtsverordnung ist für die Jahrgangsstufe 7 in Integrierten Sekundarschulen, Gemeinschaftsschulen und Gymnasien sowie für die Jahrgangsstufe 8 in Integrierten Sekundarschulen und Gemeinschaftsschulen eine Höchstgrenze von Schülerinnen und Schülern pro Lerngruppe festzulegen.

Link: https://gesetze.berlin.de/bsbe/document/ilr-SchulGBEV42P56

Schulgesetz für das Land Mecklenburg-Vorpommern (SchulG M-V)

\begin{tabular}{|l|l|}
\hline Norm & Text \\
\hline$\S 66$ & $\begin{array}{l}\text { (1) Nach dem Besuch der Grundschule entscheiden die Erziehungsberechtigten darüber, ob ihr Kind die } \\
\text { schulartunabhängige Orientierungsstufe an einer Regionalen Schule, einer Kooperativen Gesamtschule } \\
\text { oder einer Integrierten Gesamtschule besucht. Nach dem Besuch der schulartunabhängigen } \\
\text { Orientierungsstufe treffen die Erziehungsberechtigten im Rahmen der Regelungen über die Schularten }\end{array}$ \\
\hline
\end{tabular}

DDS, 113. Jg., 4(2021) 16 


\begin{tabular}{|c|c|}
\hline & $\begin{array}{l}\text { und Bildungsgänge sowie des } \S 56 \text { die Entscheidungen über den Bildungsweg ihrer Kinder. Die Schule } \\
\text { berät und unterstützt die Erziehungsberechtigten und die Schülerinnen und Schüler bei ihren } \\
\text { Entscheidungen. } \\
\text { (2) Sofern die Erziehungsberechtigten sich entgegen der Schullaufbahnempfehlung nach } \S 15 \text { Absatz } 3 \\
\text { für den Besuch des gymnasialen Bildungsganges entscheiden, gilt die Jahrgangsstufe } 7 \text { als } \\
\text { Erprobungsschuljahr. Sofern die Schülerin oder der Schüler das Erprobungsschuljahr nicht erfolgreich } \\
\text { absolviert hat, hat sie oder er diesen Bildungsgang zu verlassen. } \\
\text { (3) Die Schülerin oder der Schüler kann ab der Jahrgangsstufe } 7 \text { auf Antrag der Erziehungsberechtigten } \\
\text { in einen anderen Bildungsgang übergehen. Die Klassenkonferenz der abgebenden Schule erstellt den } \\
\text { Erziehungsberechtigten eine Empfehlung, ob aufgrund der Lernentwicklung und des Leistungsstandes } \\
\text { der Schülerin oder des Schülers eine erfolgreiche Teilnahme in derselben oder der nächsthöheren } \\
\text { Jahrgangsstufe des anderen Bildungsganges zu erwarten ist. Die Erziehungsberechtigten sind vor der } \\
\text { Empfehlung der Klassenkonferenz zu beraten. Die aufnehmende Schule hat den Übergang durch } \\
\text { geeignete Fördermaßnahmen zu erleichtern. Die aufnehmende und die abgebende Schule arbeiten bei } \\
\text { Übergängen zusammen. } \\
\text { (4) Die Klassenkonferenz kann ab der Jahrgangsstufe } 7 \text { aufgrund der Lernentwicklung und des } \\
\text { Leistungsstandes der Schülerin oder des Schülers den Erziehungsberechtigten den Wechsel in einen } \\
\text { anderen Bildungsgang empfehlen. } \\
\text { (5) Volljährige Schülerinnen und Schüler entscheiden anstelle ihrer Erziehungsberechtigten selbst. } \\
\text { (6) Bei der Aufnahme einer Schülerin oder eines Schülers aus einem anderen Land ist von der } \\
\text { Jahrgangs- und Kurseinstufung des anderen Landes auszugehen. } \\
\text { Link: https://www.landesrecht-mv.de/bsmv/document/Ilr-SchulGMV2010V22P66 }\end{array}$ \\
\hline \multicolumn{2}{|r|}{ z für das Land Nordrhein-Westfalen (NRW - SchulG) } \\
\hline & Text \\
\hline $\begin{array}{l}\S 11 \\
\text { Abs. } 5\end{array}$ & $\begin{array}{l}\text { (5) Die Grundschule erstellt mit dem Halbjahreszeugnis der Klasse } 4 \text { auf der Grundlage des } \\
\text { Leistungsstands, der Lernentwicklung und der Fähigkeiten der Schülerin oder des Schülers eine zu } \\
\text { begründende Empfehlung für die Schulform, die für die weitere schulische Förderung geeignet erscheint. } \\
\text { Ist ein Kind nach Auffassung der Grundschule für eine weitere Schulform mit Einschränkungen geeignet, } \\
\text { wird auch diese mit dem genannten Zusatz benannt. Die Eltern entscheiden nach Beratung durch die } \\
\text { Grundschule über den weiteren Bildungsgang ihres Kindes in der Sekundarstufe I. } \\
\text { Link: https://bass.schul-welt.de/6043.htm\#1-1p11 }\end{array}$ \\
\hline$\S 13$ & $\begin{array}{l}\text { (1) In der Hauptschule, der Realschule und im Gymnasium werden jeweils die Klassen } 5 \text { und } 6 \text { als } \\
\text { Erprobungsstufe geführt. } \\
\text { (2) Die Erprobungsstufe dient der Erprobung, Förderung und Beobachtung der Schülerinnen und } \\
\text { Schüler, um in Zusammenarbeit mit den Eltern die Entscheidung über die Eignung der Schülerinnen und } \\
\text { Schüler für die gewählte Schulform sicherer zu machen. } \\
\text { (3) Am Ende der Erprobungsstufe entscheidet die Klassenkonferenz, ob die Schülerin oder der Schüler } \\
\text { den Bildungsgang in der gewählten Schulform fortsetzen kann. Nach jedem Schulhalbjahr in der } \\
\text { Erprobungsstufe befindet sie außerdem darüber, ob sie den Eltern leistungsstarker Schülerinnen und } \\
\text { Schüler der Hauptschule einen Wechsel ihres Kindes zur Realschule oder zum Gymnasium und den } \\
\text { Eltern leistungsstarker Schülerinnen und Schüler der Realschule einen Wechsel ihres Kindes zum } \\
\text { Gymnasium empfiehlt. } \\
\text { (4) Im Schuljahr } 2020 / 2021 \text { findet Absatz } 3 \text { Satz } 1 \text { keine Anwendung. } \\
\text { Link: https://bass.schul-welt.de/6043.htm\#1-1p13 }\end{array}$ \\
\hline
\end{tabular}

Schulgesetz für das Land Sachsen-Anhalt (SchulG LSA) und Verordnung über die Übergänge zwischen den Schulformen in der Sekundarstufe I

\begin{tabular}{|c|c|}
\hline Norm & Text \\
\hline $\begin{array}{l}\text { \$ } 34 \\
\text { SchulG } \\
\text { LSA }\end{array}$ & $\begin{array}{l}\text { (1) Die Erziehungsberechtigten haben im Rahmen der Regelungen des Bildungsweges die Wahl } \\
\text { zwischen den Schulformen und Bildungsgängen, die zur Verfügung stehen. Volljährige Schülerinnen } \\
\text { und Schüler wählen selbst. Die Schule berät bei der Wahl des Bildungsweges. } \\
\text { (2) Nach dem } 4 \text {. Schuljahrgang wählen die Erziehungsberechtigten entsprechend den Neigungen und } \\
\text { Fähigkeiten ihrer Kinder den weiteren Bildungsgang. } \\
\text { (3) Ein Wechsel der Bildungsgänge oder Schulformen in der Sekundarstufe I kann von der Erfüllung } \\
\text { bestimmter Leistungsvoraussetzungen abhängig gemacht werden. Die Schule ist verpflichtet, } \\
\text { Schülerinnen und Schüler nach einem Wechsel des Bildungsganges oder der Schulform besonders zu } \\
\text { fördern. } \\
\text { (4) Die Aufnahme in die Schulen der Sekundarstufe II kann davon abhängig gemacht werden, dass die } \\
\text { Schülerin oder der Schüler einen bestimmten Abschluss oder berufliche Erfahrungen nachweist. Dies } \\
\text { gilt nicht für die Aufnahme in die Berufsschule. } \\
\text { (5) Eine Schülerin oder ein Schüler kann den nächsthöheren Schuljahrgang erst besuchen, wenn die } \\
\text { Klassenkonferenz entschieden hat, dass von ihnen eine erfolgreiche Mitarbeit in diesem Schuljahrgang } \\
\text { erwartet werden kann (Versetzung). Zwischen einzelnen Schuljahrgängen kann von dem Erfordernis der } \\
\text { Versetzung abgesehen werden. } \\
\text { (6) Schülerinnen und Schüler der Sekundarstufe I des Gymnasiums sollen bei der zweiten } \\
\text { Nichtversetzung an einen geeigneten Bildungsgang überwiesen werden, wenn die Klassenkonferenz } \\
\text { beschließt, dass ein erfolgreicher Besuch des Gymnasiums nicht erwartet werden kann. Schülerinnen }\end{array}$ \\
\hline
\end{tabular}




\begin{tabular}{|c|c|}
\hline & $\begin{array}{l}\text { und Schüler des } 5 \text {. Schuljahrganges sollen bereits überwiesen werden, wenn eine erfolgreiche Mitarbeit } \\
\text { auch nach einer Wiederholung nicht erwartet werden kann. } \\
\text { (7) Wer nach zwei Wiederholungen in der Sekundarschule erneut nicht versetzt wird, soll, sofern die } \\
\text { Vollzeitschulpflicht erfüllt ist, an einen geeigneten beruflichen Bildungsgang verwiesen werden. Erfolgt } \\
\text { im Fall einer nicht möglichen Versetzung die Zuweisung in einen höheren Schuljahrgang, so ist diese } \\
\text { Zuweisung im Sinne von Satz } 1 \text { als Wiederholung anzurechnen. } \\
\text { (8) Durch Prüfungen können Nichtschülerinnen und Nichtschüler die Abschlüsse der allgemeinbildenden } \\
\text { Schulen und der berufsbildenden Schulen erreichen. } \\
\text { Link: https://www.landesrecht.sachsen-anhalt.de/bst/document/ilr-SchulGST2018pP34 }\end{array}$ \\
\hline $\begin{array}{l}\text { §1 } \\
\text { Sek-I- } \\
\text { Üg-VO } \\
\text { LSA }\end{array}$ & $\begin{array}{l}\text { Gemäß } \S 34 \text { Abs. } 1 \text { des Schulgesetzes des Landes Sachsen-Anhalt haben die Erziehungsberechtigten } \\
\text { im Rahmen der Regelungen des Bildungsweges die Wahl zwischen den Schulformen, die zur Verfügung } \\
\text { stehen. Die Verordnung gilt nicht an Schulen mit gemäß } \S 5 \text { Abs. } 1 \text { Satz } 3, \S 5 a \text { Abs. } 2 \text { Satz } 2 \text { und } \S 6 \\
\text { Abs. } 1 \text { Satz } 3 \text { des Schulgesetzes des Landes Sachsen-Anhalt von der obersten Schulbehörde } \\
\text { genehmigten inhaltlichen Schwerpunkten sowie an Förderschulen. } \\
\text { Link: } \\
\text { anhalt.de/fileadmin/Bibliothek/Landesjournal/Bildung und Wissenschaft/Verordnungen//Verordnung u } \\
\text { eber die UEbergaenge zwischen den Schulformen in der Sekundarstufe I.pdf }\end{array}$ \\
\hline
\end{tabular}

\section{Sozialgesetzbuch (SGB)}

\begin{tabular}{|l|l|}
\hline Norm & Text \\
\hline § 35a & (1) Kinder oder Jugendliche haben Anspruch auf Eingliederungshilfe, wenn \\
Abs. 1 & 1. $\quad$ ihre seelische Gesundheit mit hoher Wahrscheinlichkeit länger als sechs Monate von dem für \\
SGB & ihr Lebensalter typischen Zustand abweicht, und \\
VIII & $\begin{array}{l}\text { daher ihre Teilhabe am Leben in der Gesellschaft beeinträchtigt ist oder eine solche } \\
\text { Beeinträchtigung zu erwarten ist. }\end{array}$ \\
& $\begin{array}{l}\text { Von einer seelischen Behinderung bedroht im Sinne dieser Vorschrift sind Kinder oder Jugendliche, bei } \\
\text { denen eine Beeinträchtigung ihrer Teilhabe am Leben in der Gesellschaft nach fachlicher Erkenntnis mit } \\
\text { hoher Wahrscheinlichkeit zu erwarten ist. § 27 Absatz 4 gilt entsprechend. } \\
\text { Link: https://www.gesetze-im-internet.de/sgb 8/ 35a.html }\end{array}$ \\
\hline
\end{tabular}

Strafgesetzbuch (StGB)

\begin{tabular}{|l|l|}
\hline Norm & Text \\
\hline$\S 171$ & $\begin{array}{l}\text { Wer seine Fürsorge- oder Erziehungspflicht gegenüber einer Person unter sechzehn Jahren gröblich } \\
\text { verletzt und dadurch den Schutzbefohlenen in die Gefahr bringt, in seiner körperlichen oder psychischen }\end{array}$ \\
& $\begin{array}{l}\text { Entwicklung erheblich geschädigt zu werden, einen kriminellen Lebenswandel zu führen oder der } \\
\text { Prostitution nachzugehen, wird mit Freiheitsstrafe bis zu drei Jahren oder mit Geldstrafe bestraft. } \\
\text { Link: https://www.gesetze-im-internet.de/stgb/ 171.html }\end{array}$ \\
\hline
\end{tabular}

Thüringer Schulgesetz (ThürSchulG) und Thüringer Schulordnung (ThürSchulO)

\begin{tabular}{|c|c|}
\hline Norm & Text \\
\hline $\begin{array}{l}\text { §7 } \\
\text { Abs. } 2 \\
\text { Thür } \\
\text { SchulG }\end{array}$ & $\begin{array}{l}\text { (2) Voraussetzung für den Übertritt in das Gymnasium ist eine bestandene Aufnahmeprüfung in Form } \\
\text { eines Probeunterrichts. Die Aufnahmeprüfung ist nicht bestanden, wenn sie ergibt, dass der Schüler für } \\
\text { den Besuch des Gymnasiums offensichtlich ungeeignet ist. Ein Schüler ist dann nicht geeignet, wenn } \\
\text { nach seiner Befähigung und Leistung aufgrund einer pädagogischen Prognose eine erfolgreiche } \\
\text { Teilnahme am Unterricht im Gymnasium nicht erwartet werden kann. Einer Aufnahmeprüfung bedarf es } \\
\text { nicht, wenn bei einem Schüler aufgrund des Erreichens bestimmter Leistungsvoraussetzungen in } \\
\text { einzelnen Fächern oder des Vorliegens einer auf seinen bisherigen Leistungen, seinem } \\
\text { Leistungsvermögen und seiner Leistungsbereitschaft beruhenden Empfehlung für den Bildungsweg des } \\
\text { Gymnasiums eine erfolgreiche Mitarbeit am Gymnasium erwartet werden kann. § } 17 \text { Abs. } 4 \text { bleibt } \\
\text { unberührt. Sowohl für die Aufnahmeprüfung in Form des Probeunterrichts als auch für die weitere } \\
\text { Unterrichtsteilnahme ist sicherzustellen, dass förder- bzw. unterstützungsbedürftige Schüler im Sinne } \\
\text { des gemeinsamen Unterrichts nach } \S 2 \text { Abs. } 2 \text { Satz } 2 \text { und } \S 8 \text { a alle notwendigen } \\
\text { Unterstützungsmaßnahmen und Hilfsmittel erhalten, die sie zu einer erfolgreichen Teilnahme am } \\
\text { gemeinsamen Unterricht benötigen. } \\
\text { Link: https://landesrecht.thueringen.de/bsth/documentj/lr-SchulGTH2003V12P7 }\end{array}$ \\
\hline $\begin{array}{l}125 \\
\text { Thür } \\
\text { SchulO }\end{array}$ & $\begin{array}{l}\text { (1) Voraussetzung für den Übertritt von der Grundschule und der Regelschule in die Klassenstufen } 5 \text { bis } \\
7 \text { sowie von der Gemeinschaftsschule in die Klassenstufen } 5 \text { bis } 9 \text { des Gymnasiums ist eine bestandene } \\
\text { Aufnahmeprüfung. Einer Aufnahmeprüfung bedarf es nicht, wenn der Schüler } \\
\text { 1. die in Absatz } 2 \text { geforderten Leistungsvoraussetzungen erfüllt oder } \\
\text { 2. eine Empfehlung der Klassenkonferenz für den Bildungsweg des Gymnasiums erhält. } \\
\text { (2) Leistungsvoraussetzung für den Übertritt ist, dass der Schüler im Zeugnis zum Schulhalbjahr } \\
\text { 1. der Klassenstufe } 4 \text { der Grundschule oder der Gemeinschaftsschule in den Fächern Deutsch, } \\
\text { Mathematik sowie Heimat- und Sachkunde oder } \\
\text { 2. der Klassenstufen } 5 \text { und } 6 \text { der Regelschule in den Fächern Deutsch, Mathematik und erste } \\
\text { Fremdsprache } \\
\text { jeweils mindestens die Note 'gut' erreicht hat. Ein Schüler der Klassenstufe } 7 \text { der Gemeinschaftsschule } \\
\text { muss im Zeugnis zum Schulhalbjahr in den Fächern Deutsch, Mathematik und erste Fremdsprache auf } \\
\text { der abschlussbezogenen Anspruchsebene II mindestens die Note 'gut' erreicht haben; ein Schüler der }\end{array}$ \\
\hline
\end{tabular}




\begin{tabular}{|c|c|}
\hline & 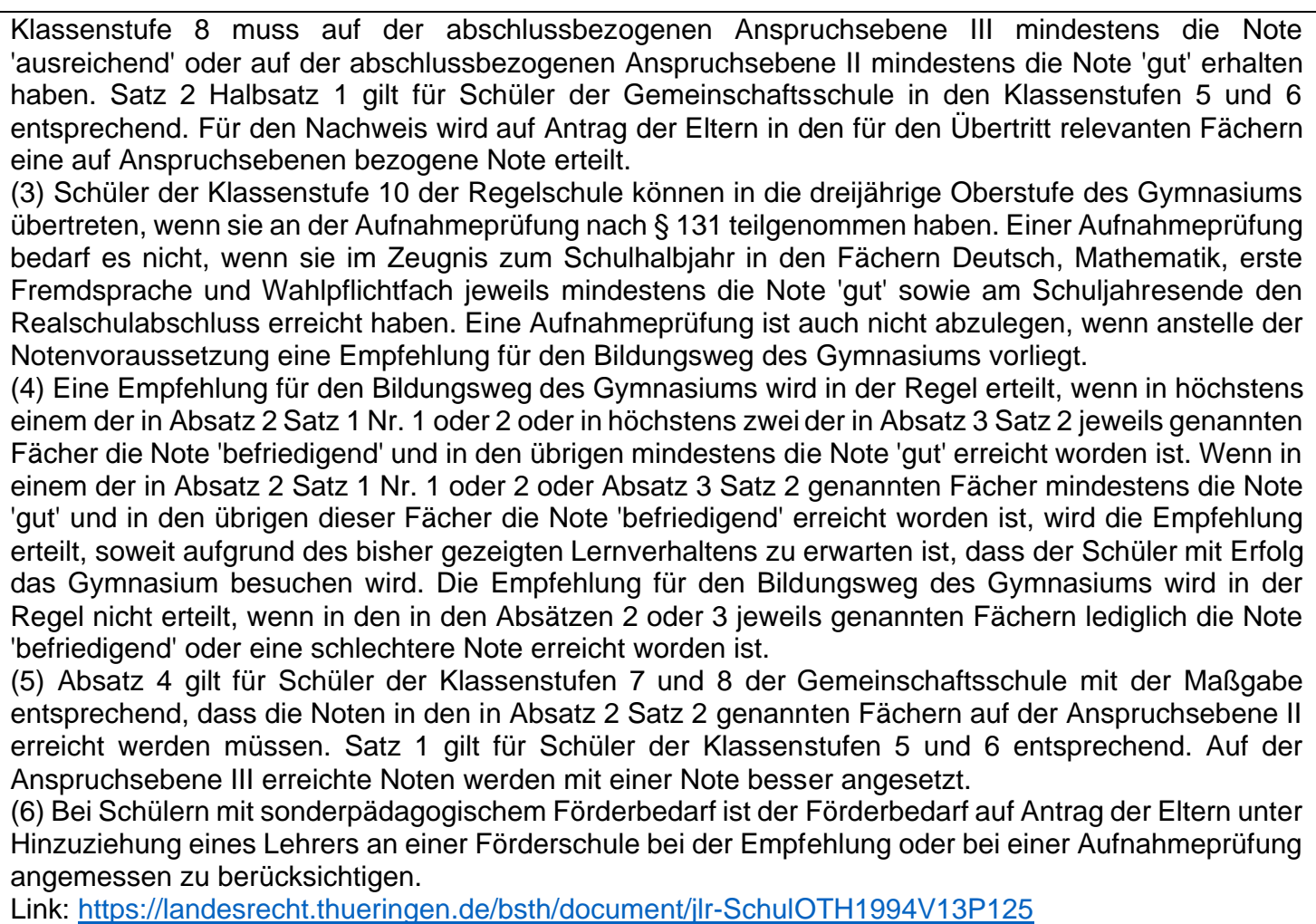 \\
\hline $\begin{array}{l}\S 131 \\
\text { Thür } \\
\text { SchulO }\end{array}$ & $\begin{array}{l}\text { (1) Eine Aufnahmeprüfung findet auf Antrag der Eltern für Schüler statt, die nicht nach } \$ 125 \text { Abs. } 1 \text { Satz } \\
2 \text { oder Abs. } 3 \text { Satz } 2 \text { und } 3 \text { von der Aufnahmeprüfung befreit sind. Die Aufnahmeprüfung kann in der } \\
\text { Regel nicht nachgeholt werden. } \\
\text { (2) Das zuständige Schulamt bestimmt die Gymnasien, die die Aufnahmeprüfung durchführen. Die } \\
\text { Aufnahmeprüfung besteht aus Probeunterricht an drei aufeinanderfolgenden Tagen mit jeweils vier } \\
\text { Unterrichtsstunden. Der Probeunterricht erfolgt in einzelnen Fächern oder fächerübergreifend. } \\
\text { (3) Die Inhalte für den Probeunterricht werden von einem Gymnasium des Schulamtsbereichs für die } \\
\text { Aufnahmeprüfungen in diesem Schulamtsbereich vorbereitet. } \\
\text { (4) Die Prüfungskommission wird vom zuständigen Schulamt bestellt und besteht aus zwei Lehrern, die } \\
\text { am Gymnasium unterrichten, und einem Lehrer der Schulart Grundschule oder Gemeinschaftsschule für } \\
\text { den Übertritt in die Klassenstufe } 5 \text { oder einem Lehrer der Schularten Regelschule oder } \\
\text { Gemeinschaftsschule für den Übertritt in die Klassenstufen } 6,7,10 \text { und } 11 \text { oder einem Lehrer der } \\
\text { Schulart Gemeinschaftsschule für den Übertritt in die Klassenstufen } 8 \text { und } 9 \text {. Das Schulamt bestimmt } \\
\text { einen der in Satz } 1 \text { genannten Lehrer zum Vorsitzenden der Prüfungskommission. Der Probeunterricht } \\
\text { in den einzelnen Unterrichtsstunden wird von einem Mitglied der Prüfungskommission durchgeführt; die } \\
\text { übrigen Mitglieder sind als Beobachter tätig. } \\
\text { (5) Die Prüfungskommission setzt am Ende der Aufnahmeprüfung das Ergebnis fest; es lautet } \\
\text { "bestanden" oder "nicht bestanden". Die Aufnahmeprüfung ist nicht bestanden, wenn die } \\
\text { Prüfungskommission einstimmig festgestellt hat, daß der Schüler für den Besuch des Gymnasiums } \\
\text { offensichtlich ungeeignet ist. } \\
\text { (6) Über die Aufnahmeprüfung wird ein Protokoll angefertigt, das den wesentlichen Verlauf der Prüfung, } \\
\text { Unterrichtsbeobachtungen und das Prüfungsergebnis enthält. } \\
\text { Link: https://landesrecht.thueringen.de/bsth/document/IIr-SchulOTH1994V38P131 }\end{array}$ \\
\hline
\end{tabular}

Übergreifende Schulordnung Rheinland-Pfalz ÜSchulO Rh.-Pf.

Link:

https://bm.rlp.de/fileadmin/bm/Publikationen_BM/Dateien_Publikationen/Broschu re Schulordnung Internet 20 20.pdf

\begin{tabular}{|c|c|}
\hline Norm & Text \\
\hline $\begin{array}{l}\S 12 \\
\text { Abs. } 2\end{array}$ & $\begin{array}{l}\text { (2) Die Eltern entscheiden auf der Grundlage der } \\
\text { Empfehlung der Grundschule, welche Schulart ihr } \\
\text { Kind besuchen soll. }\end{array}$ \\
\hline$\S 20$ & $\begin{array}{l}\text { (1) Am Ende der Orientierungsstufe erhalten die Schülerinnen und Schüler einer Realschule plus oder } \\
\text { eines Gymnasiums, denen ein Wechsel der Schullaufbahn zu raten ist, sowie alle Schülerinnen und } \\
\text { Schüler einer schulartübergreifenden Orientierungsstufe eine Empfehlung der Klassenkonferenz für den } \\
\text { Besuch der Realschule plus oder des Gymnasiums. Den Eltern ist zuvor Gelegenheit zu einem Gespräch } \\
\text { zu geben. } \\
\text { (2) Grundlage der Schullaufbahnempfehlung sind das Lernverhalten und die Leistungen unter } \\
\text { besonderer Berücksichtigung der Entwicklung der Schülerin oder des Schülers in der Orientierungsstufe. }\end{array}$ \\
\hline
\end{tabular}




\begin{tabular}{|l|l|}
\hline & $\begin{array}{l}\text { Die Schule entscheidet im Benehmen mit dem Schulelternbeirat über die Empfehlungsmaßstäbe. Sie } \\
\text { werden den Eltern zu Beginn der Orientierungsstufe bekannt gegeben. } \\
\text { (3) Eine Empfehlung der Realschule plus für das Gymnasium kann nur ausgesprochen werden, wenn } \\
\text { der Durchschnitt der Leistungen in den Fächern Deutsch, Mathematik und erste Fremdsprache sowie } \\
\text { der Durchschnitt der Leistungen in den übrigen Fächern mindestens 2,5 beträgt. Bei der Ermittlung des } \\
\text { Durchschnitts bleibt eine zweite Dezimalstelle unberücksichtigt. Sofern Schülerinnen und Schülern der } \\
\text { Realschule plus keine Empfehlung für das Gymnasium ausgesprochen wurde, können sie eine Prüfung } \\
\text { gemäß § 21 ablegen. } \\
{[\ldots]}\end{array}$ \\
\hline$\S 21$ & $\begin{array}{l}\text { (1) Die Prüfung gliedert sich in eine schriftliche und, sofern es zur Sicherung der Entscheidung } \\
\text { erforderlich ist, in eine mündliche Prüfung. Die schriftliche Prüfung besteht aus einer Aufgabe schriftlicher }\end{array}$ \\
$\begin{array}{l}\text { Sprachgestaltung von 90 Minuten im Fach Deutsch sowie in einem schriftlichen Leistungsnachweis in } \\
\text { der ersten Fremdsprache und in Mathematik von jeweils 45 Minuten. Die mündliche Prüfung soll in jedem } \\
\text { der drei Fächer zehn Minuten nicht überschreiten. Die Prüfung findet am fünften und sechsten }\end{array}$ \\
$\begin{array}{l}\text { Unterrichtstag vor den Sommerferien statt. } \\
\text { (2) Die Prüfung ist mit Erfolg abgelegt, wenn der Gesamtdurchschnitt der Noten der drei Prüfungsfächer } \\
\text { mindestens 2,5 beträgt }\end{array}$ \\
\hline$\S 30$ & $\begin{array}{l}\text { (1) Wer an der Integrierten Gesamtschule oder der Realschule plus den qualifizierten } \\
\text { Sekundarabschluss I und eine Berechtigung zum Besuch der gymnasialen Oberstufe erworben hat, wird } \\
\text { in die gymnasiale Oberstufe aufgenommen. Die Anmeldung soll zum 1. März mit dem Halbjahreszeugnis } \\
\text { erfolgen. Anmelden kann sich, wer im Halbjahreszeugnis die Berechtigungsvoraussetzungen erfüllt; } \\
\text { andernfalls kann eine Anmeldung auch unverzüglich nach Erhalt des Abschlusszeugnisses erfolgen. } \\
{[\ldots]}\end{array}$ \\
\hline
\end{tabular}

UN-Behindertenrechtskonvention (UN-BRK)

Link: https://www.behindertenbeirat-

muenchen.de/images/stories/downloadarchiv/Basisdokumente/UN Konvention deutsch.pdf

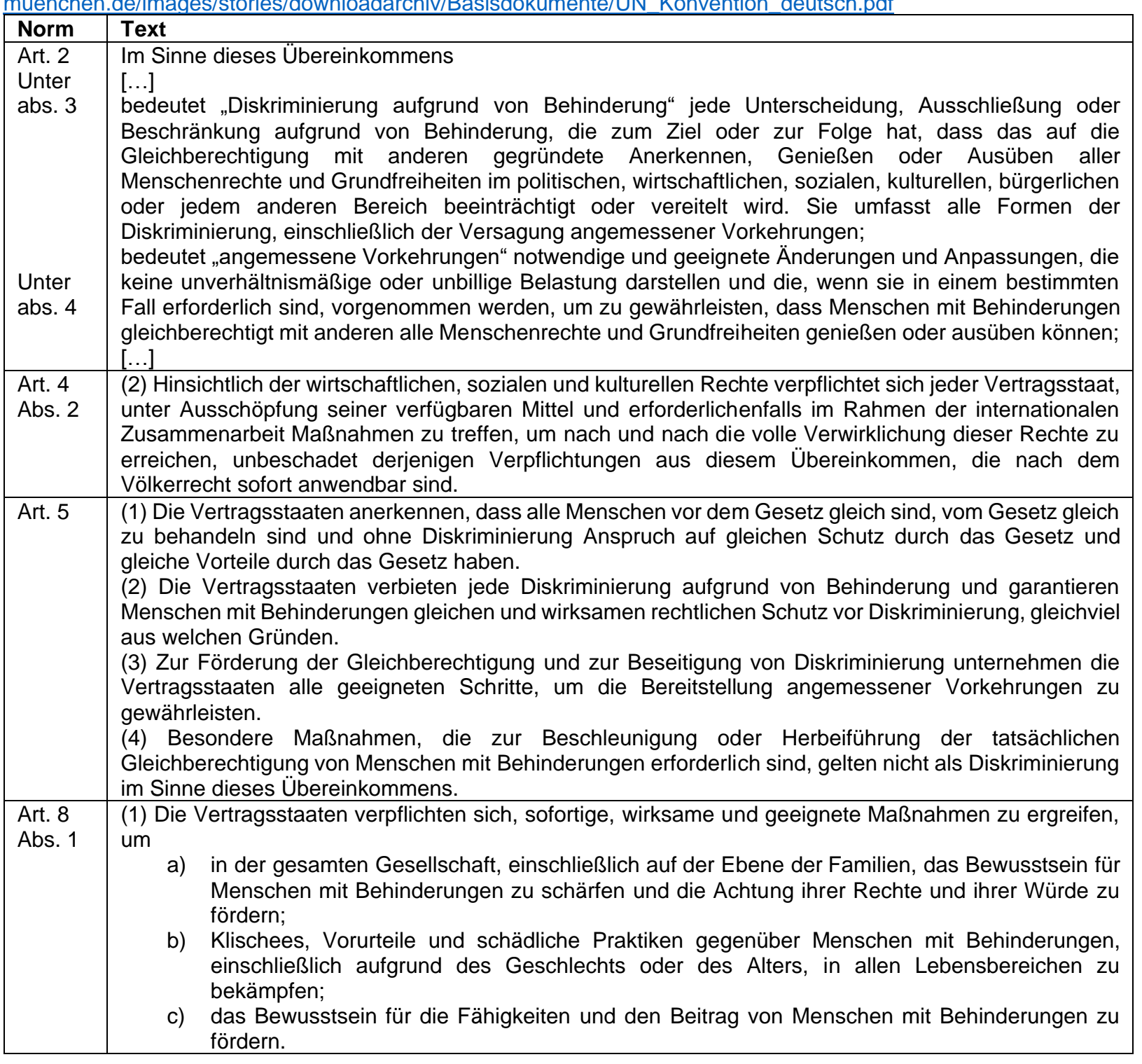

DDS, 113. Jg., 4(2021) 20 


\begin{tabular}{|c|c|}
\hline $\begin{array}{l}\text { Art. } 9 \\
\text { Abs. } 1\end{array}$ & $\begin{array}{l}\text { (1) Um Menschen mit Behinderungen eine unabhängige Lebensführung und die volle Teilhabe in allen } \\
\text { Lebensbereichen zu ermöglichen, treffen die Vertragsstaaten geeignete Maßnahmen mit dem Ziel, für } \\
\text { Menschen mit Behinderungen den gleichberechtigten Zugang zur physischen Umwelt, zu } \\
\text { Transportmitteln, Information und Kommunikation, einschließlich Informations- und } \\
\text { Kommunikationstechnologien und -systemen, sowie zu anderen Einrichtungen und Diensten, die der } \\
\text { Öffentlichkeit in städtischen und ländlichen Gebieten offenstehen oder für sie bereitgestellt werden, zu } \\
\text { gewährleisten. Diese Maßnahmen, welche die Feststellung und Beseitigung von Zugangshindernissen } \\
\text { und -barrieren einschließen, gelten unter anderem für } \\
\text { a) Gebäude, Straßen, Transportmittel sowie andere Einrichtungen in Gebäuden und im Freien, } \\
\text { einschließlich Schulen, Wohnhäusern, medizinischer Einrichtungen und Arbeitsstätten; } \\
\text { b) Informations-, Kommunikations- und andere Dienste, einschließlich elektronischer Dienste und } \\
\text { Notdienste. }\end{array}$ \\
\hline Art. 19 & $\begin{array}{l}\text { Die Vertragsstaaten dieses Übereinkommens anerkennen das gleiche Recht aller Menschen mit } \\
\text { Behinderungen, mit gleichen Wahlmöglichkeiten wie andere Menschen in der Gemeinschaft zu leben, } \\
\text { und treffen wirksame und geeignete Maßnahmen, um Menschen mit Behinderungen den vollen Genuss } \\
\text { dieses Rechts und ihre volle Einbeziehung in die } 16 \text { Gemeinschaft und Teilhabe an der Gemeinschaft } \\
\text { zu erleichtern, indem sie unter anderem gewährleisten, dass } \\
\text { a) Menschen mit Behinderungen gleichberechtigt die Möglichkeit haben, ihren Aufenthaltsort zu } \\
\text { wählen und zu entscheiden, wo und mit wem sie leben, und nicht verpflichtet sind, in } \\
\text { besonderen Wohnformen zu leben; } \\
\text { b) Menschen mit Behinderungen Zugang zu einer Reihe von gemeindenahen } \\
\text { Unterstützungsdiensten zu Hause und in Einrichtungen sowie zu sonstigen gemeindenahen } \\
\text { Unterstützungsdiensten haben, einschließlich der persönlichen Assistenz, die zur } \\
\text { Unterstützung des Lebens in der Gemeinschaft und der Einbeziehung in die Gemeinschaft } \\
\text { sowie zur Verhinderung von Isolation und Absonderung von der Gemeinschaft notwendig ist; } \\
\text { c) gemeindenahe Dienstleistungen und Einrichtungen für die Allgemeinheit Menschen mit } \\
\text { Behinderungen auf der Grundlage der Gleichberechtigung zur Verfügung stehen und ihren } \\
\text { Bedürfnissen Rechnung tragen. }\end{array}$ \\
\hline 24 & 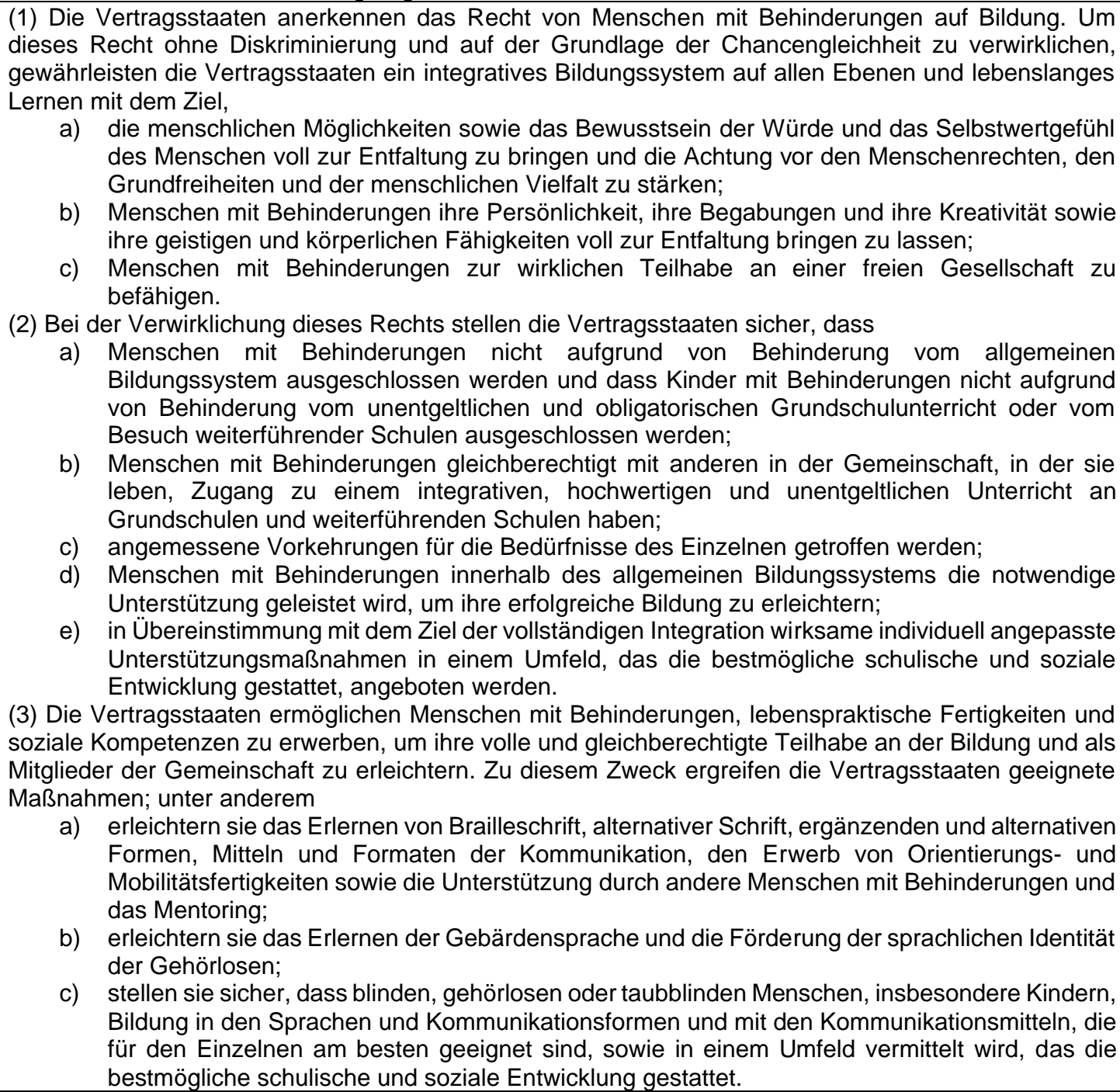 \\
\hline
\end{tabular}




\begin{tabular}{|c|c|}
\hline & $\begin{array}{l}\text { (4) Um zur Verwirklichung dieses Rechts beizutragen, treffen die Vertragsstaaten geeignete } \\
\text { Maßnahmen zur Einstellung von Lehrkräften, einschließlich solcher mit Behinderungen, die in } \\
\text { Gebärdensprache oder Brailleschrift ausgebildet sind, und zur Schulung von Fachkräften sowie } \\
\text { Mitarbeitern und Mitarbeiterinnen auf allen Ebenen des Bildungswesens. Diese Schulung schließt die } \\
\text { Schärfung des Bewusstseins für Behinderungen und die Verwendung geeigneter ergänzender und } \\
\text { alternativer Formen, Mittel und Formate der Kommunikation sowie pädagogische Verfahren und } \\
\text { Materialien zur Unterstützung von Menschen mit Behinderungen ein. } \\
\text { (5) Die Vertragsstaaten stellen sicher, dass Menschen mit Behinderungen ohne Diskriminierung und } \\
\text { gleichberechtigt mit anderen Zugang zu allgemeiner Hochschulbildung, Berufsausbildung, } \\
\text { Erwachsenenbildung und lebenslangem Lernen haben. Zu diesem Zweck stellen die Vertragsstaaten } \\
\text { sicher, dass für Menschen mit Behinderungen angemessene Vorkehrungen getroffen werden. }\end{array}$ \\
\hline Art. 50 & $\begin{array}{l}\text { Der arabische, der chinesische, der englische, der französische, der russische und der spanische } \\
\text { Wortlaut dieses Übereinkommens sind gleichermaßen verbindlich. Zu Urkund dessen haben die } \\
\text { unterzeichneten, von ihren Regierungen hierzu gehörig befugten Bevollmächtigten dieses } \\
\text { Übereinkommen unterschrieben. }\end{array}$ \\
\hline
\end{tabular}

UN-Kinderrechtskonvention (UN-KRK)

\begin{tabular}{|c|c|}
\hline Norm & Text \\
\hline Art. 28 & $\begin{array}{l}\text { (1) Die Vertragsstaaten erkennen das Recht des Kindes auf Bildung an; um die Verwirklichung dieses } \\
\text { Rechts auf der Grundlage der Chancengleichheit fortschreitend zu erreichen, werden sie insbesondere } \\
\text { a) den Besuch der Grundschule für alle zur Pflicht und unentgeltlich machen; } \\
\text { b) die Entwicklung verschiedener Formen der weiterführenden Schulen allgemeinbildender und } \\
\text { berufsbildender Art fördern, sie allen Kindern verfügbar und zugänglich machen und geeignete } \\
\text { Maßnahmen wie die Einführung der Unentgeltlichkeit und die Bereitstellung finanzieller } \\
\text { Unterstützung bei Bedürftigkeit treffen; } \\
\text { c) allen entsprechend ihren Fähigkeiten den Zugang zu den Hochschulen mit allen geeigneten } \\
\text { Mitteln ermöglichen; } \\
\text { d) Bildungs- und Berufsberatung allen Kindern verfügbar und zugänglich machen; e) Maßnahmen } \\
\text { treffen, die den regelmäßigen Schulbesuch fördern und den Anteil derjenigen, welche die } \\
\text { Schule vorzeitig verlassen, verringern. } \\
\text { (2) Die Vertragsstaaten treffen alle geeigneten Maßnahmen, um sicherzustellen, dass die Disziplin in } \\
\text { der Schule in einer Weise gewahrt wird, die der Menschenwürde des Kindes entspricht und im Einklang } \\
\text { mit diesem Übereinkommen steht. } \\
\text { (3) Die Vertragsstaaten fördern die internationale Zusammenarbeit im Bildungswesen, insbesondere um } \\
\text { zur Beseitigung von Unwissenheit und Analphabetentum in der Welt beizutragen und den Zugang zu } \\
\text { wissenschaftlichen und technischen Kenntnissen und modernen Unterrichtsmethoden zu erleichtern. } \\
\text { Dabei sind die Bedürfnisse der Entwicklungsländer besonders zu berücksichtigen. } \\
\text { Link: https://www.unicef.de/informieren/ueber-uns/fuer-kinderrechte/un-kinderrechtskonvention }\end{array}$ \\
\hline
\end{tabular}

Verfassung des Freistaats Bayern (BayVerf)

\begin{tabular}{|l|l|}
\hline Norm & Text \\
\hline Art. 129 & $\begin{array}{l}\text { (1) Alle Kinder sind zum Besuch der Volksschule und der Berufsschule verpflichtet. } \\
\text { (2) Der Unterricht an diesen Schulen ist unentgeltlich. } \\
\text { Link: https://www.gesetze-bayern.de/Content/Document/BayVerf-129 }\end{array}$ \\
\hline
\end{tabular}

Verfassung des Freistaats Sachsen (SächsLV)

\begin{tabular}{|l|l|}
\hline Norm & Text \\
\hline Art. 7 & (2) Das Land bekennt sich zur Verpflichtung der Gemeinschaft, alte und behinderte Menschen zu \\
Abs. 2 & $\begin{array}{l}\text { unterstützen und auf die Gleichwertigkeit ihrer Lebensbedingungen hinzuwirken. } \\
\text { Link: https://www.revosax.sachsen.de/vorschrift/3975-Saechsische-Verfassung\#a7 }\end{array}$ \\
\hline
\end{tabular}

Verwaltungsgerichtsordnung (VwGO)

\begin{tabular}{|l|l|}
\hline Norm & Text \\
\hline$\S 4$ & $\begin{array}{l}\text { (1) Durch Klage kann die Aufhebung eines Verwaltungsakts (Anfechtungsklage) sowie die Verurteilung } \\
\text { zum Erlaß eines abgelehnten oder unterlassenen Verwaltungsakts (Verpflichtungsklage) begehrt } \\
\text { werden. } \\
\text { (2) Soweit gesetzlich nichts anderes bestimmt ist, ist die Klage nur zulässig, wenn der Kläger geltend } \\
\text { macht, durch den Verwaltungsakt oder seine Ablehnung oder Unterlassung in seinen Rechten verletzt } \\
\text { zu sein. } \\
\text { Link: http://www.gesetze-im-internet.de/vwgo/ 42.html }\end{array}$ \\
\hline $\begin{array}{l}\text { (1) Das Oberverwaltungsgericht entscheidet im Rahmen seiner Gerichtsbarkeit auf Antrag über die } \\
\text { Gültigkeit } \\
1 . \quad \text { von Satzungen, die nach den Vorschriften des Baugesetzbuchs erlassen worden sind, sowie } \\
\quad \text { von Rechtsverordnungen auf Grund des } \S 246 \text { Abs. 2 des Baugesetzbuchs }\end{array}$ \\
$\begin{array}{l}\text { 2. von anderen im Rang unter dem Landesgesetz stehenden Rechtsvorschriften, sofern das } \\
\text { (2) Dandesrecht dies bestimmt. Antrag kann jede natürliche oder juristische Person, die geltend macht, durch die } \\
\text { Rechtsvorschrift oder deren Anwendung in ihren Rechten verletzt zu sein oder in absehbarer Zeit verletzt }\end{array}$ \\
\hline
\end{tabular}


zu werden, sowie jede Behörde innerhalb eines Jahres nach Bekanntmachung der Rechtsvorschrift stellen. Er ist gegen die Körperschaft, Anstalt oder Stiftung zu richten, welche die Rechtsvorschrift erlassen hat. Das Oberverwaltungsgericht kann dem Land und anderen juristischen Personen des öffentlichen Rechts, deren Zuständigkeit durch die Rechtsvorschrift berührt wird, Gelegenheit zur Äußerung binnen einer zu bestimmenden Frist geben. $\S 65$ Abs. 1 und 4 und $\S 66$ sind entsprechend anzuwenden.

(2a) (weggefallen)

(3) Das Oberverwaltungsgericht prüft die Vereinbarkeit der Rechtsvorschrift mit Landesrecht nicht, soweit gesetzlich vorgesehen ist, daß die Rechtsvorschrift ausschließlich durch das Verfassungsgericht eines Landes nachprüfbar ist.

(4) Ist ein Verfahren zur Überprüfung der Gültigkeit der Rechtsvorschrift bei einem Verfassungsgericht anhängig, so kann das Oberverwaltungsgericht anordnen, daß die Verhandlung bis zur Erledigung des Verfahrens vor dem Verfassungsgericht auszusetzen sei.

(5) Das Oberverwaltungsgericht entscheidet durch Urteil oder, wenn es eine mündliche Verhandlung nicht für erforderlich hält, durch Beschluß. Kommt das Oberverwaltungsgericht zu der Überzeugung, daß die Rechtsvorschrift ungültig ist, so erklärt es sie für unwirksam; in diesem Fall ist die Entscheidung allgemein verbindlich und die Entscheidungsformel vom Antragsgegner ebenso zu veröffentlichen wie die Rechtsvorschrift bekanntzumachen wäre. Für die Wirkung der Entscheidung gilt § 183 entsprechend. (6) Das Gericht kann auf Antrag eine einstweilige Anordnung erlassen, wenn dies zur Abwehr schwerer Nachteile oder aus anderen wichtigen Gründen dringend geboten ist. Link: http://www.gesetze-im-internet.de/vwgo/ 47.html 